University of Warwick institutional repository: http://go.warwick.ac.uk/wrap This paper is made available online in accordance with publisher policies. Please scroll down to view the document itself. Please refer to the repository record for this item and our policy information available from the repository home page for further information.

To see the final version of this paper please visit the publisher's website. Access to the published version may require a subscription.

Author(s): JON WARREN and DAVID WILLIAMS Article Title: PROBABILISTIC STUDY OF A DYNAMICAL SYSTEM

Year of publication: 2000

Link to published version:

http://dx.doi.org/10.1112/S0024611500012594

Publisher statement: None 


\title{
PROBABILISTIC STUDY OF A DYNAMICAL SYSTEM
}

\author{
JON WARREN and DAVID WILLIAMS
}

[Received 2 August 1999; revised 18 October 1999]

\section{Introduction and summary}

This paper investigates the relation between a branching process and a non-linear dynamical system in $\mathbb{C}^{2}$. This idea has previously been fruitful in many investigations, including that of the FKPP equation by McKean, Neveu, Bramson, and others. Our concerns here are somewhat different from those in other work: we wish to elucidate those features of the dynamical system which correspond to the long-term behaviour of the random process. In particular, we are interested in how the dimension of the global attractor corresponds to that of the tail $\sigma$-algebra of the process. The Poincaré-Dulac operator which (locally) intertwines the nonlinear system with its linearization may sometimes be exhibited as a FourierLaplace transform of tail-measurable random variables; but things change markedly when parameters cross values giving the 'primary resonance' in the Poincaré-Dulac sense. Probability proves effective in establishing global properties amongst which is a clear description of the global convergence to the attractor. Several of our probabilistic results are analogues of ones obtained by Kesten and Stigum, and by Athreya and Ney, for discrete branching processes. Our simpler context allows the use of Itô calculus.

Because the paper bridges two subjects, dynamical-system theory and probability theory, we take considerable care with the exposition of both aspects. For probabilist readers, we provide a brief guide to Poincaré-Dulac theory; and we take the view that in a paper which we hope will be read by analysts, it would be wrong to fudge any details of rigour in our probabilistic arguments.

\subsection{An example}

Before we begin, consider the flow $\varphi_{t} \circ z(0)=z(t)$ of the dynamical system $\dot{z}=2 z-2 z^{2}$ on $\mathbb{C}$, and the 'linear' flow $\mathscr{L}_{t}$ associated with the linearization $\dot{w}=2 w$ of $\varphi$ at 0 . These flows are globally isomorphic if considered on the Riemann sphere in that if we write $\Gamma w:=w /(1+w)$ with inverse map $\gamma z:=z /(1-z)$, then $\varphi_{t} \circ \Gamma=\Gamma \circ \mathscr{L}_{t}$ for every $t$. (Of course, ':=' means 'is defined to equal'.) If $E$ denotes the set $\{z: \Re(z) \geqslant 0\}$, then $\left(\varphi_{t}: t \geqslant 0\right)$ acts on $E$, and $\mathscr{A}:=\bigcap_{t \geqslant 0} \varphi_{t} E$ is the disc $\overline{\mathbb{D}}\left(\frac{1}{2}, \frac{1}{2}\right):=\left\{z:\left|z-\frac{1}{2}\right| \leqslant \frac{1}{2}\right\}$. Thus, the set $\mathscr{A}$ is homeomorphic to the unit ball in $\mathbb{R}^{2}$. Moreover, $\mathscr{A}$ consists of the fixed points 0 and 1 of $\varphi$ together with the tracks of heteroclinic orbits of $\varphi$ (which are arcs of circles) going from 0 to 1 within $E$. (A heteroclinic orbit going from 0 to 1 within $E$ is a path of the form $\left\{\left(t, \varphi_{t} z\right): t \in \mathbb{R}\right\}$ where $\varphi_{t} z \in E(t \in \mathbb{R}), \varphi_{t} z \rightarrow 0$ as $t \rightarrow-\infty$ and $\varphi_{t} z \rightarrow 1$ as $t \rightarrow \infty$; its track is then the subset $\left\{\varphi_{t} z: t \in \mathbb{R}\right\}$ of $E$.) 
But, if we step up a 'complex' dimension, things are already much more interesting.

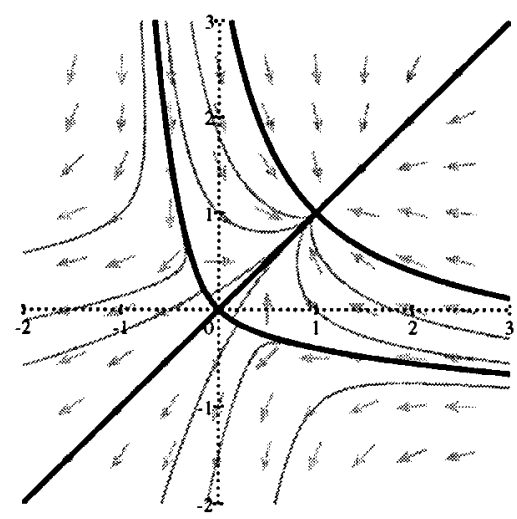

Flow on $\mathbb{R}^{2}$,

3 coincident fixed points' at $(0,0)$

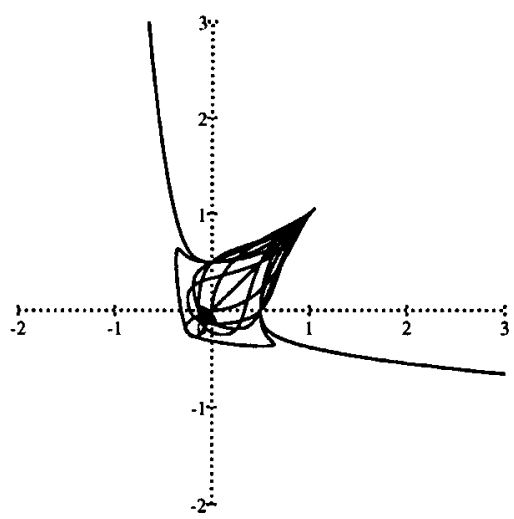

Some $\left(\Re z_{1}(\cdot), 9 z_{2}(\cdot)\right)$ projections of orbits in $\mathbb{C}^{2}$

Figure 1. Flows associated with (1.1.1) when $q_{1}=q_{2}=1$.

Here is a hint of things to come, but in an example where extra structure causes dramatic simplification. Let $q_{1}$ and $q_{2}$ be positive real constants. Consider solutions $\left\{\left(z_{1}(t), z_{2}(t)\right):-\infty<t<\infty\right\}$, where (for $\left.n=1,2\right) z_{n}: \mathbb{R} \rightarrow \mathbb{C}$, of

$$
\begin{aligned}
& \dot{z}_{1}=2 z_{1}-2 z_{1}^{2}+q_{1}\left(z_{2}-z_{1}\right), \\
& \dot{z}_{2}=2 z_{2}-2 z_{2}^{2}+q_{2}\left(z_{1}-z_{2}\right) .
\end{aligned}
$$

Figure 1 illustrates the (somewhat 'freaky') case when $q_{1}=q_{2}=1$, the lefthand half showing the flow of (1.1.1) on $\mathbb{R}^{2}$, and the right-hand half showing the $\left(z_{1}, z_{2}\right) \mapsto\left(\Re z_{1}, \mathfrak{R} z_{2}\right)$ projection of some orbits in $\mathbb{C}^{2}$.

Restrict attention now to solutions for which

$$
\Re\left(z_{n}(t)\right) \geqslant 0 \quad(n=1,2,-\infty<t<\infty) .
$$

Clearly the set $\mathscr{A}_{t}$ of all possible values of $\left(z_{1}(t), z_{2}(t)\right)$ for such solutions is independent of $t: \mathscr{A}_{t}=\mathscr{A}$. If we have 'strong coupling' in the sense that $q_{1}+q_{2} \geqslant 1$, then, as we shall see at the end of $\S 3$,

$$
z_{1}(t)=z_{2}(t)=z(t) \quad(\text { say }) \quad(-\infty<t<\infty),
$$

with $z(\cdot)$ satisfying our previous $\dot{z}=2 z-2 z^{2}$, so that

$$
\mathscr{A}=\left\{\left(z_{1}, z_{2}\right): z_{1}=z_{2} \in \overline{\mathbb{D}}\left(\frac{1}{2}, \frac{1}{2}\right)\right\}
$$

and $\mathscr{A}$ is homeomorphic to the unit ball in $\mathbb{R}^{2}$. For 'weak coupling' with $q_{1}+q_{2}<1, \mathscr{A}$ is homeomorphic to the unit ball in $\mathbb{R}^{3}$. Figure 2 , discussed further at the end of $\S 1.3$, illustrates the (non-freaky) case when $q_{1}=q_{2}=0.15$. If we had allowed the 'no coupling' case when $q_{1}=q_{2}=0$, then, in that case, we would have $\mathscr{A}=\overline{\mathbb{D}}\left(\frac{1}{2}, \frac{1}{2}\right)^{2}$, and $\mathscr{A}$ would be homeomorphic to the unit ball in $\mathbb{R}^{4}$. We are interested in how these things (and, of course, other deeper analytic results) relate to probability theory. 


\subsection{The dynamical system on $E$ and its 'dual' Markov process $X$}

Let $I=\{1,2\}$, and for $i \in I$, let $r_{i}$ and $q_{i}$ in $(0, \infty)$ be strictly positive numbers. For $z:[0, \infty) \times I \rightarrow \mathbb{C}$, we consider the system

$$
\begin{aligned}
& \frac{d z_{1}}{d t}=q_{1}\left(z_{2}-z_{1}\right)+r_{1} z_{1}-2 z_{1}^{2}, \\
& \frac{d z_{2}}{d t}=q_{2}\left(z_{1}-z_{2}\right)+r_{2} z_{2}-2 z_{2}^{2} .
\end{aligned}
$$

We shall use column-vector notation for $\mathbb{C}$-valued functions on $I$, rewriting (1.2.1) as

$$
\dot{z}:=\frac{d z}{d t}=(R+Q) z-2 z^{2},
$$

where

$$
R:=\left(\begin{array}{cc}
r_{1} & 0 \\
0 & r_{2}
\end{array}\right), \quad Q:=\left(\begin{array}{rr}
-q_{1} & q_{1} \\
q_{2} & -q_{2}
\end{array}\right) .
$$

A study of dynamical systems in $\mathbb{C}^{2}$ has been made by Hille [6]. His interests are rather different from ours.

By sticking to the 2-(complex-)dimensional case, we avoid excessive notation and can illustrate some aspects with pictures. Everything extends in a natural way to the case when $I=\{1,2, \ldots, n\}$ and $Q$ is a matrix, with positive off-diagonal elements and zero row sums, which acts as a self-adjoint operator on $L^{2}(I, \mu)$ for some measure $\mu$ on $I$.

Analytical results on the dynamical system (1.2.1) begin in the next subsection. We now prepare for the use of probabilistic methods utilizing a "measure-valued process', one hardly worthy of the name because it can equally well be viewed as a process on $\mathbb{R}^{2}$ ! However, the 'measure-valued' terminology is the right one for all contexts.

Let $\mathscr{M}(I)$ denote the set of (non-negative) measures $x$ on $I$; we can think of $x \in \mathscr{M}(I)$ as a row vector $\left(x^{(1)}, x^{(2)}\right)$. For $x \in \mathscr{M}(I)$ and a function $f$ on $I$, we write $x(f)$ or $x f$ for the 'integral' $x^{(1)} f_{1}+x^{(2)} f_{2}$.

The reason that probability theory may be applied to classical potential theory is that if $f$ is a harmonic function on $\mathbb{R}^{n}$, and $B=\left\{B_{t}: t \geqslant 0\right\}$ is a Brownian motion on $\mathbb{R}^{n}$, then $f(B)=\left\{f\left(B_{t}\right): t \geqslant 0\right\}$ is a local martingale. What allows us to apply probability theory to our dynamical system is that there is an $\mathscr{M}(I)$ valued process $X=\left\{X_{t}: t \geqslant 0\right\}=\{X(t): t \geqslant 0\}$ with the property that if $z \in \mathbb{C}^{2}$ is such that $\varphi_{s} z$ is finite for $0 \leqslant s \leqslant t$, then

is a local martingale.

$$
\left\{\exp \left(-X_{s} \varphi_{t-s} z\right): 0 \leqslant s \leqslant t\right\}
$$

Here is an intuitive description of an approximation $\left\{X_{N}(t): t \geqslant 0\right\}$ to the process $\{X(t): t \geqslant 0\}$ started from a point $x$ in $\mathscr{M}(I), N$ being a large positive integer. Start with $x^{(i)} N$ particles each of mass $N^{-1}$ at each state $i$ in $I$. (Pretend that each $x^{(i)} N$ is an integer!) 'Independently of everything else', a particle at state $i$ at time $t \geqslant 0$ will within a small time interval $[t, t+h]$, either

jump to the other state of $I$ with probability $q_{i} h+o(h)$, or die (and have mass zero) with probability $2 N h+o(h)$, or 
split into two particles (each of mass $N^{-1}($ !)) with probability $\left(r_{i}+2 N\right) h+o(h)$, or

remain as it is.

We write $X_{N}^{(i)}(t) N$ for the number of (alive) particles at state $i$ at time $t$. Suppose that we know the values $X_{N}^{(i)}(t) N(i \in I)$ for some $t$. Then (ignoring $o(h)$ terms), over the time-interval $[t, t+h]$, the number of particles at state 1 will increase by the sum of $X_{N}^{(1)}(t) N$ random variables each taking the values

$-1,1,0$ with probabilities $\left(q_{1}+2 N\right) h,\left(r_{1}+2 N\right) h, 1-\left(4 N+r_{1}+q_{1}\right) h$, and $X_{N}^{(2)}(t) N$ random variables each taking the values

1,0 with respective probabilities $q_{2} h, 1-q_{2} h$,

all summands being independent. By elementary probability theory, the increase in total mass of particles at state 1 during $[t, t+h]$ (given $X_{N}(t)$ ) will have mean and variance respectively

$$
\begin{aligned}
& h\left\{X_{N}^{(1)}(t)\left(-q_{1}+r_{1}\right)+X_{N}^{(2)}(t) q_{2}\right\}+N^{-1} o(h), \\
& h\left\{4 X_{N}^{(1)}(t)\right\}+N^{-1} h\left\{X_{N}^{(1)}(t)\left(q_{1}+r_{1}\right)+X_{N}^{(2)}(t) q_{2}\right\}+N^{-1} o(h) .
\end{aligned}
$$

We can therefore imagine that as $N \rightarrow \infty$, the process $X_{N}(\cdot)$ will converge to a weak solution of the stochastic differential equation (SDE):

$$
d X_{t}=d B_{t} \sigma\left(X_{t}\right)+d t X_{t}(R+Q), \quad X_{0}=x,
$$

where

$$
\sigma\left(X_{t}\right)=\operatorname{diag}\left(2 \sqrt{X_{t}^{(1)}}, 2 \sqrt{X_{t}^{(2)}}\right)
$$

and $B$ is a (row-vector) Brownian motion on $\mathbb{R}^{2}$.

One normally formulates the study of weak solutions of SDEs in terms of martingale problems. The reader is asked to take for granted the result from the theory of measure-valued processes (see Dawson [5]) described in the next paragraph. The remainder of the probability theory in the paper is standard martingale material as found, for example, in books by Revuz and Yor [10] or Rogers and Williams [12]. Especially, the following martingale-problem result is exactly equivalent to the statement that equation (1.2.3) has a weak solution unique in law. The solution $X$ therefore has the strong Markov property.

For any $x \in \mathscr{M}(I)$, there exists an $\mathscr{M}(I)$-valued process $X=\left\{X_{t}: t \geqslant 0\right\}$ with $X_{0}=x$ and such that for any function $f$ on $I$,

$$
U_{t}^{f}:=X_{t} f-\int_{0}^{t} X_{s}(R+Q) f d s \text { defines a local martingale }
$$

(relative to the augmented natural filtration of $X$ ) with quadratic-variation process

$$
\left[U^{f}\right]_{t}=4 \int_{0}^{t} X_{s}\left(f^{2}\right) d s
$$

and that the law $\mathbb{P}^{x}$ of $X$ started at $x$ is uniquely specified by these properties: we say that $X$ is an $(R, Q, x)$-process. We let $\mathbb{E}^{x}$ denote the expectation associated with $\mathbb{P}^{x}$.

If we say that a statement holds almost surely, or a.s. for short, we mean that for each $x \in \mathscr{M}(I)$, it holds with $\mathbb{P}^{x}$ probability 1 . If we say that a process is a martingale, we mean that for each $x$, the process is a $\mathbb{P}^{x}$-martingale relative to the 
$\mathbb{P}^{x}$-augmented natural filtration of $X$. And so on. (Markov aficionados will sweeten to taste.) In a statement of the form $\mathbb{E}^{x}(\cdot)=\mathbb{E}^{x}(\cdot)$, true for every $x$, we shall often drop the superscript $x$.

We define

$$
\zeta:=\inf \left\{t: X_{t}=(0,0)\right\} \leqslant \infty
$$

The total-mass process $X_{t}(1)$ of $X$ satisfies

$$
d X_{t}(1)=2 \sqrt{X_{t}(1)} d \beta+X_{t}(r) d t,
$$

where $r=R \mathbf{1}$ and, by a familiar Pythagorean property, $\beta$ is a Brownian motion. We compare the $X_{t}(1)$-process with the squared ' $\delta$-dimensional' Bessel process $Z$ which satisfies

$$
d Z=2 \sqrt{Z} d \beta+\delta d t .
$$

See $\S$ XI.1 of $[\mathbf{1 0}]$, or $\S$ V.48 of [12]. If $0<\delta<2$, then $Z$ has positive probability of hitting 0; and it follows from the Yamada-Ikeda-Watanabe Comparison Theorem [12, Theorem V.43.1] that for any compact neighbourhood $F$ of $(0,0)$ in $[0, \infty)^{2}$, there exists $\varepsilon>0$ such that, for every $x \in F, \mathbb{P}^{x}(\zeta<1) \geqslant \varepsilon$. (For a more significant use of the Comparison Theorem spelt out in detail, see $\S 4.1$.) It is therefore almost surely true that if there is a (random) sequence of times $t$ with $t \rightarrow \infty$ such that $X_{t} \in F$ for every $t$ in that sequence, then $\zeta<\infty$ for that realization. (See the end of this subsection for a precise form of this intuitive argument.) It now follows that

$$
\text { almost surely, either } \zeta<\infty \text { or } X_{t}(1) \rightarrow \infty \text {, }
$$

each alternative having positive probability when $X_{0}(1) \neq 0$.

THE KEY ADDITIVE PROPERTY 1.2.1. If $X$ is an $(R, Q, x)$-process and $Y$ is an $(R, Q, y)$-process independent of $X$, then $X+Y$ is an $(R, Q, x+y)$-process.

This is intuitively obvious from the 'particle' picture, and is easily deduced from equation (1.2.3) or from the martingale-problem formulation.

EXTINCTION PROBABILITY PROPERTIES 1.2.2. On combining the two properties just described, we conclude that for $0<t \leqslant \infty$, for some real $e_{t}$ in $(0, \infty)^{2}$,

$$
\mathbb{P}^{x}(\zeta<t)=\exp \left\{-x\left(e_{t}\right)\right\} \quad(x \in \mathscr{M}(I)) .
$$

Moreover, for some $e_{\infty}$ in $(0, \infty)^{2}$,

$$
\mathbb{P}^{x}(\zeta<\infty)=\exp \left\{-x\left(e_{\infty}\right)\right\}
$$

As promised, we now explain how result (1.2.6) may be proved rigorously.

Let $F_{k}=[0, k] \times[0, k]$. Then there exists $\varepsilon_{k}>0$ such that for $x \in F_{k}$, $\mathbb{P}^{x}(\zeta<1) \geqslant \varepsilon_{k}$. Define

$$
T_{n, k}:=\inf \left\{t: t \geqslant T_{n-1, k}+1, X_{t} \in F_{k}\right\} \leqslant \infty .
$$

Then $\left\{T_{n-1, k}<\zeta\right\} \in \mathscr{F}\left(T_{n-1, k}\right)$, where $\{\mathscr{F}(t)\}$ is the augmented natural filtration of $X$, whence, by the Strong Markov Property, we have for any $x$, and 
for $n \geqslant 2$,

$$
\begin{aligned}
\mathbb{P}^{x}\left[T_{n, k}<\zeta\right] & =\mathbb{P}^{x}\left[T_{n, k}<\zeta ; T_{n-1, k}<\zeta\right] \\
& =\mathbb{E}^{x}\left[\mathbb{P}^{x}\left(T_{n, k}<\zeta \mid \mathscr{F}\left(T_{n-1, k}\right)\right) ; T_{n-1, k}<\zeta\right] \\
& =\mathbb{E}^{x}\left[\mathbb{P}^{X\left(T_{n-1, k}\right)}\left(T_{1, k}<\zeta\right) ; T_{n-1, k}<\zeta\right] \\
& \leqslant\left(1-\varepsilon_{k}\right) \mathbb{P}^{x}\left[T_{n-1, k}<\zeta\right]
\end{aligned}
$$

whence,

$$
\mathbb{P}^{x}\left[T_{n, k}<\zeta\right] \leqslant\left(1-\varepsilon_{k}\right)^{n-1} \quad \text { and } \quad \mathbb{P}^{x}\left(\bigcap_{n}\left\{T_{n, k}<\zeta\right\}\right)=0 .
$$

Hence

$$
\mathbb{P}^{x}\left[\zeta<\infty ; X_{t}(1) \nrightarrow \infty\right]=\mathbb{P}\left(\bigcup_{k} \bigcap_{n}\left\{T_{n, k}<\zeta\right\}\right)=0
$$

\subsection{The flow on the global attractor}

The matrix $R+Q$ has two distinct real eigenvalues: $\lambda_{0}$, the larger (PerronFrobenius) one, which is strictly positive with a strictly positive associated eigenfunction (right eigenvector) $u$, and the smaller $\lambda_{1}$ which may take either sign and which has a real eigenfunction $v$ with $v_{1}$ and $v_{2}$ of opposite signs. To summarize our notation:

$$
\begin{array}{ll}
(R+Q) u=\lambda_{0} u & \left(\lambda_{0}>0, u>0\right), \\
(R+Q) v=\lambda_{1} v & \left(\lambda_{0}>\lambda_{1}\right) .
\end{array}
$$

We normalize the right eigenfunctions $u$ and $v$ and a (Perron-Frobenius) left eigenmeasure (row-vector) $m$ of $R+Q$ associated with $\lambda_{0}$, whence $m(R+Q)=m \lambda_{0}$, so as to satisfy:

$$
m(u)=1, \quad m\left(u^{2}\right)=1, \quad m\left(v^{2}\right)=1, \quad m(v)=0,
$$

the last being inevitable. We note that $v^{2}=u+\rho v$ for some $\rho \in \mathbb{R}$.

The system (1.2.2) defines a flow $\varphi=\left(\varphi_{t}: t \geqslant 0\right)$, via $\varphi_{t}(z(0))=z(t)$, on the set

$$
E:=\left\{\left(z_{1}, z_{2}\right) \in \mathbb{C}^{2}: \Re\left(z_{i}\right) \geqslant 0 \text { for } i=1,2\right\} ;
$$

this is clear because the vector field of $\varphi$ on the boundary of $E$ never points 'outwards'. It is often $E$ rather than $\mathbb{C}^{2}$ which we shall consider as the 'underlying universe'. When we work with $\mathbb{C}^{2}$ as universe, we always have to bear in mind that solutions of (1.2.1) can explode in finite time. Thus the 'flow' $\varphi_{t}$ on $\mathbb{C}^{2}$ will only be defined 'locally'. That no explosion can occur for the flow on $E$ will follow from the probabilistic study.

Define the maximal invariant subset $\mathscr{A}$ of $E$ by

$$
\mathscr{A}:=\bigcap_{t \geqslant 0} \varphi_{t} E .
$$

We shall provide a probabilistic proof of the following theorem, exhibiting the main part of it as a consequence of the Riemann-Lebesgue Lemma. The points $e_{\infty}$ and $e_{t}$ in the theorem have the simple probabilistic significance already described. 
THEOREM 1.3.1. The set $\mathscr{A}$ consists of the origin, the unique other fixed point $e_{\infty}$ of $\varphi$ within $E$, and the tracks of heteroclinic orbits of $\varphi$ lying within $E$ which go from $(0,0)$ to $e_{\infty}$. We define

$$
\mathscr{A}^{-}:=\mathscr{A} \backslash\left\{e_{\infty}\right\}
$$

Let $\bar{E}=E \cup \infty_{E}$ denote the one-point compactification of $E$. Then each $\varphi_{t}$ extends to a continuous map (still denoted by $\varphi_{t}$ ) on $\bar{E}$ and

$$
e_{t}:=\varphi_{t}\left(\infty_{E}\right) \rightarrow e_{\infty} \quad \text { as } t \rightarrow \infty .
$$

(Thus, by any time $t>0$, the infinite part of the boundary of $E$ is collapsed to a single point.) It is now obvious that $\mathscr{A}$ is a global attractor for $E$ in the strong sense that

$$
\sup _{z \in E} \inf _{a \in \mathscr{A}}\left\|\varphi_{t} z-a\right\| \rightarrow 0 \quad \text { as } t \rightarrow \infty .
$$

(The principle involved in the last statement is that if $H_{n}$ is a decreasing sequence of compact sets with $\bigcap H_{n}=H$ and if $N$ is an open neighbourhood of $H$, then, for some $n_{0}, H_{n} \subseteq N$ for $n \geqslant n_{0}$. For if not, then $\left\{H_{n} \cap N^{\mathrm{c}}: n \in \mathbb{N}\right\}\left(N^{\mathrm{c}}\right.$ being the complement of $N$ ) is a sequence of compact sets with the finiteintersection property, whence $H \cap N^{\mathrm{c}}=\bigcap\left(H_{n} \cap N^{\mathrm{c}}\right) \neq \emptyset$, a contradiction. For the application, take $H_{n}=\varphi_{n} \bar{E}, H=\mathscr{A}$, and $N=\{z: d(z, \mathscr{A})<\varepsilon\}$.)

We are interested in the topology of $\mathscr{A}$, and in the convergence of $\varphi_{t} E$ to $\mathscr{A}$.

The linearized version of $(1.2 .1)$ at the fixed point $(0,0)$ is given by

$$
\frac{d z}{d t}=(R+Q) z
$$

We shall denote by $\left(\mathscr{L}_{t}: t \in \mathbb{R}\right)$ the 'linear' flow on $\mathbb{C}^{2}$ for (1.3.6); thus,

$$
\mathscr{L}_{t} w=e^{(R+Q) t} w, \quad \text { for } w \in \mathbb{C}^{2} .
$$

The analysis of $\mathscr{A}$ leads us to separate out these cases:

$$
\lambda_{0}<2 \lambda_{1}, \quad \lambda_{0}>2 \lambda_{1}, \quad \lambda_{0}=2 \lambda_{1} \text { (the 'primary resonance'), }
$$

though for some purposes the last two 'amalgamate'. Define a cone $K$ (not compact!) in $E$ as follows:

$$
\begin{aligned}
& \text { if } \lambda_{0}<2 \lambda_{1} \text {, then } K:=\left\{\left(\alpha_{0}+\beta_{0} i\right) u+\beta_{1} i v: \alpha_{0}, \beta_{0}, \beta_{1} \in \mathbb{R}, \alpha_{0} \geqslant 0\right\} \text {, } \\
& \text { if } \lambda_{0} \geqslant 2 \lambda_{1} \text {, then } K:=\left\{\left(\alpha_{0}+\beta_{0} i\right) u: \alpha_{0}, \beta_{0} \in \mathbb{R}, \alpha_{0} \geqslant 0\right\} \text {. }
\end{aligned}
$$

The cone $K$ is invariant under the action of the linear semigroup $\mathscr{L}$, but it is not necessarily the maximal $\mathscr{L}$-invariant subset of $E$. Our next result shows that $K$ is the tangent space at the origin (in a slightly non-standard sense, since we are at the boundary of $E$ ) of $\mathscr{A}$; and moreover the dynamical systems $(K, \mathscr{L})$ and $\left(\mathscr{A}^{-}, \varphi\right)$ are isomorphic. The map $\Gamma$ is a kind of 'exponential map' from $K$ to $\mathscr{A}^{-}$. The following theorem is (as will be explained in the next section) very closely related to Poincaré's work on normal forms of differential equations, though it does have 'global' aspects.

THEOREM 1.3.2. The following statements hold.

If $\lambda_{0}<2 \lambda_{1}$, then $\mathscr{A}$ is homeomorphic to the unit ball in $\mathbb{R}^{3}$.

If $\lambda_{0} \geqslant 2 \lambda_{1}$, then $\mathscr{A}$ is homeomorphic to the unit ball in $\mathbb{R}^{2}$. 
There exists a unique homeomorphism

$$
\Gamma: K \rightarrow \mathscr{A}^{-}, \text {with inverse } \gamma: \mathscr{A}^{-} \rightarrow K,
$$

with the following properties:

(a) $\Gamma$ fixes the origin;

(b) $\Gamma$ intertwines the semigroups $\mathscr{L}$ and $\varphi$ : indeed, for all $t$ in $\mathbb{R}$,

$$
\Gamma \circ \mathscr{L}_{t}=\varphi_{t} \circ \Gamma \text { on } K
$$

(c) for each $k \in K$,

$$
\begin{aligned}
& \text { if } \lambda_{0}<2 \lambda_{1} \text {, then } \varphi_{-t} \Gamma k=\mathscr{L}_{-t} k+O\left(e^{-2 \lambda_{1} t}\right) \text { as } t \rightarrow \infty, \\
& \text { if } \lambda_{0} \geqslant 2 \lambda_{1} \text {, then } \varphi_{-t} \Gamma k=\mathscr{L}_{-t} k+O\left(e^{-2 \lambda_{0} t}\right) \text { as } t \rightarrow \infty .
\end{aligned}
$$

For $k \in K$ and $a \in \mathscr{A}^{-}$, we have

$$
\begin{aligned}
& \Gamma k=\lim _{t \rightarrow \infty} \varphi_{t} \mathscr{L}_{-t} k, \\
& \gamma a=\lim _{t \rightarrow \infty} \mathscr{L}_{t} \varphi_{-t} a .
\end{aligned}
$$

If $\bar{K}=K \cup\left\{\infty_{K}\right\}$ is the one-point compactification of $K$, then $\Gamma$ extends to a homeomorphism

$$
\Gamma: \bar{K} \rightarrow \mathscr{A},
$$

with $\Gamma$ mapping $\infty_{K}$ to $e_{\infty}$.

Probability theory gives explicit 'path-integral' formulae for $\Gamma$ and $\gamma$ from which the theorem follows quickly.

Remark 1.3.3. Moving from the $E$ to the $\mathbb{C}^{2}$ universe not only raises the possibility of explosion, but also brings in two further (possibly coincident) fixed points of $\varphi$. The relation between the orbits which go to or from these further fixed points and the probability theory is tantalizing. Orbits in $\mathbb{R}^{2}$ which link the (at most two) fixed points in $\mathbb{R}^{2} \backslash[0, \infty)^{2}$ to $e_{\infty} d o$ have probabilistic interpretations in terms of 'Doob $h$-transforms' of the underlying process $X$.

Remark 1.3.4. The set $\bigcap_{t \geqslant 0} \varphi_{t}\left([0, \infty)^{2}\right)=\mathscr{A} \cap \mathbb{R}^{2}$ is always a 1-dimensional

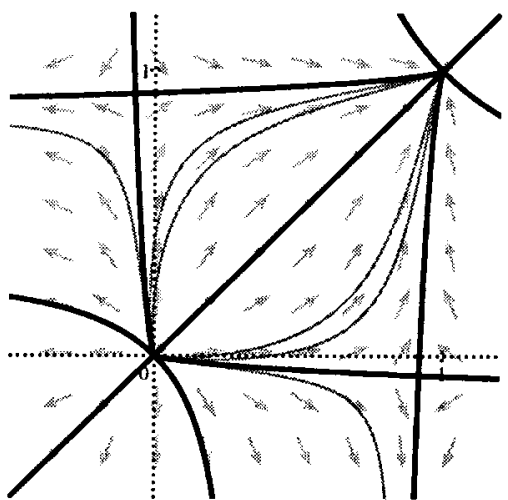

Flow on $\mathbb{R}^{2}$, with four fixed points in $\mathbb{R}^{2}$

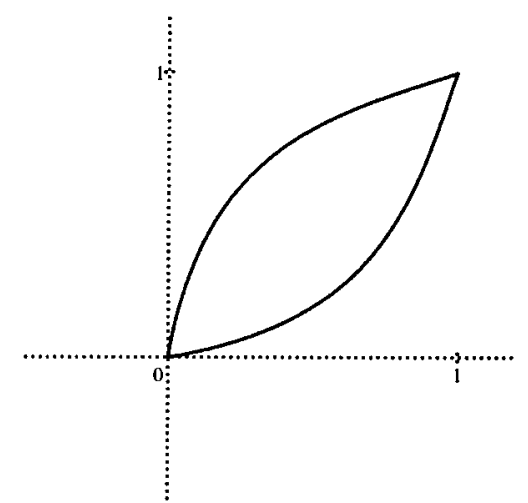

$\left(\Re z_{1}(\cdot), \Re z_{2}(\cdot)\right)$ projection of two 'extreme' orbits in $\mathscr{A}$

FIGURE 2. Flows associated with (1.1.1) when $q_{1}=q_{2}=0.15$. 

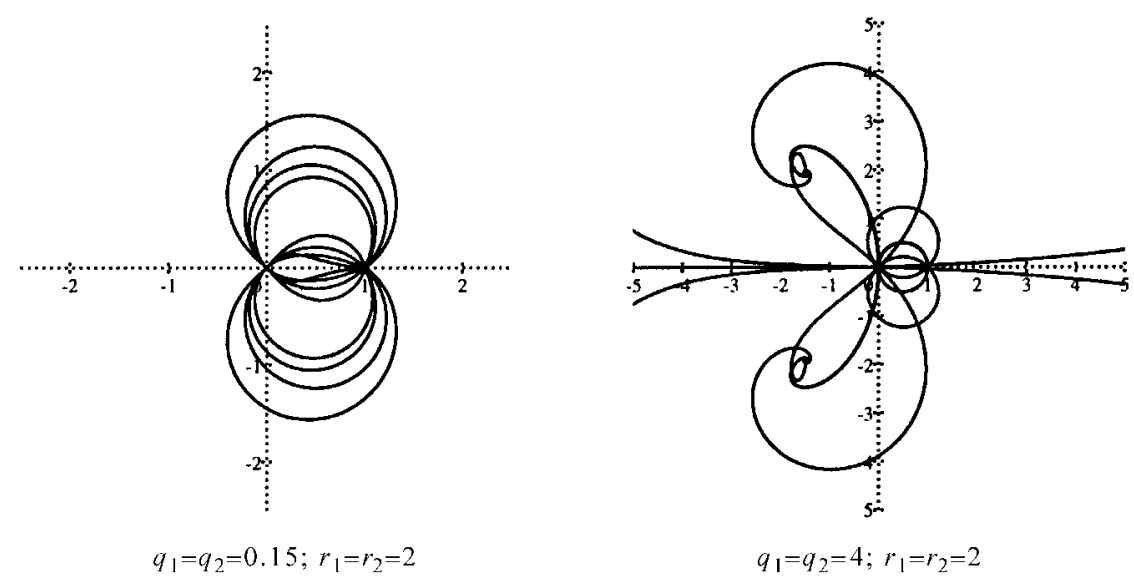

Figure 3. Some $z \mapsto z_{1}$ projections of orbits in $\mathbb{C}^{2}$.

set consisting of $(0,0), e_{\infty}$, and the unique orbit (modulo time-shift by an arbitrary constant) which links them within $[0, \infty)^{2}$. This is proved at the end of $\S 3$.

Figures 2 and 3. The left-hand part of Figure 2 shows the flow of (1.2.1) on $\mathbb{R}^{2}$ for a certain choice of the parameters. In accordance with Remark 1.3.4, all links between $(0,0)$ and $e_{\infty}=(1,1)$ in the picture, except for the straight line, exit the positive quadrant. The right-hand side of Figure 2 shows two curves which are the $\left(z_{1}, z_{2}\right) \mapsto\left(\Re z_{1}, \Re z_{2}\right)$ projections of orbits lying within $\mathscr{A}$ linking $(0,0)$ to $e_{\infty}$; of course, these two curves do not exit the first quadrant. The Poincare normal-form expansion was used to ensure that the projections of the two orbits subtend the maximal angle at $(0,0)$. This maximum angle is $2 \arctan \sqrt{1-\left(q_{1}+q_{2}\right)}$ (in our case where $r_{1}=r_{2}=2$ and $q_{1}+q_{2}<1$ ). In spite of the fact that the $\left(z_{1}, z_{2}\right) \mapsto\left(\Re z_{1}, \Re z_{2}\right)$ projection of $\mathscr{A}$ has a corner at 0 in $\mathbb{R}^{2}$ (in a sense, how could it not?), the boundary of $\mathscr{A}$ is smooth near $(0,0)$ in $\mathbb{C}^{2}$.

Figure 3 shows $z \mapsto z_{1}$ projections of orbits for two sets of parameters, the orbits having the same ' $t=0$ ' points in the two cases. The left-hand picture uses the same parameter values as Figure 2, and it mainly illustrates small perturbations (due to weak coupling) from the circular arcs of the 1-complex-dimensional system with which we began; however, it must be remembered that for these parameter values, there are real fixed points at $\left(z_{1}, z_{2}\right)=(0.92,-0.07)$ and $\left(z_{1}, z_{2}\right)=(-0.07,0.92)$ (to two places). In the right-hand picture, there are fixed points at

$$
\left(z_{1}, z_{2}\right)=\left(\frac{1}{2}(-3 \pm i \sqrt{15}), \frac{1}{2}(-3 \mp i \sqrt{15})\right),
$$

and their influence is clearly seen.

Note 1.3.5. It is worth emphasizing that for equation (1.1.1) with $q_{1}=q_{2}=q$, the primary-resonance $\left(\lambda_{0}=2 \lambda_{1}\right)$ case is when $q=\frac{1}{2}$, whereas the case of coincident fixed points at the boundary between all real and some non-real fixed points' occurs when $q=1$, the case illustrated in Figure 1. Note: this is related to the probabilistic fact that as the coupling strength is increased, the tail $\sigma$-algebra for our process 'drops in dimension' before the Martin boundary does.

Analytic results on our dynamical system continue in $\S 1.5$.

\subsection{The probabilistic significance of $\Gamma$ and $\gamma$}

THEOREM 1.4.1. The following statements hold. 
(a) The flow $\left\{\varphi_{t}: t \geqslant 0\right\}$ on $E$ may be described probabilistically via

$$
\exp \left\{-x\left(\varphi_{t} z\right)\right\}=\mathbb{E}^{x} \exp \left\{-X_{t}(z)\right\}, \quad \text { for } x \in \mathscr{M}(I),
$$

which exhibits $\varphi_{t}(z)$ as a Léry-Khintchine exponent.

(b) Let $a \in \mathscr{A}^{-}$. Then $\exp \left\{-X_{t} \varphi_{-t} a\right\}$ is a bounded martingale, and

$$
g(a):=\lim X_{t} \varphi_{-t} a \text { exists almost surely and in } L^{1} .
$$

(c) Let $k \in K$. Then $X_{t} \mathscr{L}_{-t} k$ is a martingale bounded in $L^{2}$, and

$$
G(k):=\lim X_{t} \mathscr{L}_{-t} k \text { exists almost surely and in } L^{2} .
$$

(d) The equation

$$
g(a)=G(k), \quad \text { almost surely, }
$$

describes both $\Gamma$ and $\gamma:$ for $a \in \mathscr{A}^{-}$, there is a unique $k=\gamma a \in K$ such that (1.4.4) holds; and for $k \in K$, there is a unique $a=\Gamma k \in \mathscr{A}^{-}$such that (1.4.4) holds. Thus,

$$
\begin{array}{rll}
g(a)=G(\gamma a) & \text { almost surely } & \left(a \in \mathscr{A}^{-}\right), \\
G(k)=g(\Gamma k) & \text { almost surely } & (k \in K) .
\end{array}
$$

In particular,

$$
M_{t}:=X_{t} \mathscr{L}_{-t} u=e^{-\lambda_{0} t} X_{t} u \quad \text { and } \quad N_{t}:=X_{t} \mathscr{L}_{-t} v=e^{-\lambda_{1} t} X_{t} v
$$

are martingales. The value $M_{\infty}$ always exists almost surely and in $L^{2}$. The value $N_{\infty}$ exists almost surely (and then in $L^{2}$ ) if and only if $\lambda_{0}<2 \lambda_{1}$.

In the case when $\lambda_{0}<2 \lambda_{1}$, we have the following Fourier-Laplace formula for the analytic extension of $\Gamma$ to a neighbourhood of $(0,0)$ in $\mathbb{C}^{2}:$ if $w=\xi_{0} u+\xi_{1} v$, then

$$
\exp \{-x \Gamma w\}=\mathbb{E}^{x} \exp \left\{-\xi_{0} M_{\infty}-\xi_{1} N_{\infty}\right\} .
$$

Note. Part (a) of Theorem 1.4.1 makes it clear that the flow $\left\{\varphi_{t}: t \geqslant 0\right\}$ on $E$ cannot explode. Formally, we would have equation (1.4.1) valid for $t$ less than the explosion time for $z$, etc.

\subsection{The global convergence of $\varphi_{t} E$ to the attractor $\mathscr{A}$}

Our aim now is to obtain better understanding of the convergence of $\varphi_{t} E$ to $\mathscr{A}$. The following theorem, an analytic counterpart of a probabilistic theorem of Kesten and Stigum on discrete branching processes, is the key to this.

THEOREM 1.5.1. The result

extends to a result

$$
\varphi_{t} \mathscr{L}_{-t} k \rightarrow \Gamma k \quad \text { as } t \rightarrow \infty \quad(k \in K)
$$

$$
\varphi_{t} s_{-t} z \rightarrow \Gamma(\Pi z) \quad \text { as } t \rightarrow \infty \quad(z \in E),
$$

where $s_{-t}(t \geqslant 0)$ and $\Pi$ are maps

$$
s_{-t}: E \rightarrow E \text { (onto), } \quad s_{-t}=\mathscr{L}_{-t} \text { on } K,
$$

and

$$
\text { П: } E \rightarrow K, \quad \Pi=\text { id } \text { on } K,
$$


$\Pi$ having the important additional property that

$$
z \in E \text { and } \Pi z=(0,0) \text { imply that } z=(0,0) .
$$

We use the notation for $z \in E$ :

$$
z=\left(\alpha_{0}+\beta_{0} i\right) u+\left(\alpha_{1}+\beta_{1} i\right) v \in E \quad\left(\alpha_{0}, \beta_{0}, \alpha_{1}, \beta_{1} \in \mathbb{R}\right),
$$

so that $\alpha_{0} u+\alpha_{1} v$ is non-negative. Note especially that, therefore,

$$
\alpha_{0} \geqslant 0 \text {; and if } \alpha_{0}=0 \text {, then } \alpha_{1}=0 .
$$

The maps $s_{-t}$ and $\Pi$ are defined as follows:

(a) if $\lambda_{0}<2 \lambda_{1}$, then

$$
\begin{aligned}
s_{-t}(z) & :=e^{-\lambda_{0} t}\left[\left(\alpha_{0}+\beta_{0} i\right) u+\alpha_{1} v\right]+e^{-\lambda_{1} t} \beta_{1} i v, \\
\Pi(z) & :=\left(\alpha_{0}+\beta_{0} i\right) u+\beta_{1} i v,
\end{aligned}
$$

(b) if $\lambda_{0}>2 \lambda_{1}$, then

$$
\begin{aligned}
s_{-t}(z) & :=e^{-\lambda_{0} t}\left[\left(\alpha_{0}+\beta_{0} i\right) u+\alpha_{1} v\right]+e^{-\lambda_{0} t / 2} \beta_{1} i v, \\
\Pi(z) & :=\left(\alpha_{0}+\frac{1}{2} C \beta_{1}^{2}+\beta_{0} i\right) u,
\end{aligned}
$$

(c) if $\lambda_{0}=2 \lambda_{1}$, then

$$
\begin{aligned}
s_{-t}(z) & :=e^{-\lambda_{0} t}\left[\left(\alpha_{0}+\beta_{0} i\right) u+\alpha_{1} v\right]+(1+t)^{-1 / 2} e^{-\lambda_{0} t / 2} \beta_{1} i v, \\
\Pi(z) & :=\left(\alpha_{0}+\frac{1}{2} C^{*} \beta_{1}^{2}+\beta_{0} i\right) u .
\end{aligned}
$$

Here, $C=4 /\left(\lambda_{0}-2 \lambda_{1}\right)$, and $C^{*}=4$.

We shall see later how this theorem relates to Poincaré's work. Those already familiar with resonance can see how it is reflected in (c) above.

It is the following 'punchline' which clinches the analytic appropriateness of the definitions of $s_{-t}$ and $\Pi$ and clarifies the role of $\mathscr{A}$ as a global attractor. Recall that $s_{-t}$ maps $E$ onto $E$ and that $\Gamma \circ \Pi$ maps $E$ onto $\mathscr{A}^{-}$.

THEorem 1.5.2. In Theorem 1.5.1, as $t \rightarrow \infty$,

$$
\varphi_{t} s_{-t} z \rightarrow \Gamma(\Pi z) \text { uniformly over } z \in E \text {. }
$$

\subsection{The tail $\sigma$-algebra $\mathscr{T}$ of $X$}

One of the hardest problems for the probability and/or the analysis is to characterize the tail $\sigma$-algebra $\mathscr{T}$ of the process $X$. Warren's thesis [13] proves that 'modulo null sets', $\mathscr{T}=\sigma\left(M_{\infty}, N_{\infty}\right)$ when both $M_{\infty}$ and $N_{\infty}$ exist. The thesis conjectures that when $N_{\infty}$ fails to exist, then, 'modulo null sets', $\mathscr{T}=\sigma\left(M_{\infty}\right)$, and proves this when $r_{1}=r_{2}$. (An additional assumption that $q_{1}=q_{2}$ may be dropped without changing Warren's argument.)

There are numerous interesting questions on tail $\sigma$-algebras, Martin boundaries, etc. However, these relate to the study of the parabolic equation

$$
\left(\frac{\partial}{\partial t}-\mathscr{G}\right) f=0
$$


where $\mathscr{G}$ is the infinitesimal generator of $X$; see $\S 4.1$ below. Quite different techniques are required for that study; and we defer discussion to later papers.

\section{Remarks on the 'normal form' of equation (1.2.1)}

In order to relate the probability theory to the analysis of normal forms of differential equations, we shall, in this section only, assume that we are in the so-called Poincaré regime, that is, for our case, that $(0,0)$ is a source in that $\lambda_{1}>0$, whence

$$
\lambda_{0}>\lambda_{1}>0
$$

\subsection{Poincaré(-Dulac) theory}

We give a brief 'practical' summary of this theory for the reader's convenience. See Chapter 5 and later chapters of Arnold [1] for further discussion.

We wish to study solutions of

$$
\dot{z}(t):=\frac{d z}{d t}=(R+Q) z-2 z^{2}
$$

for a $\mathbb{C}^{2}$-valued function $z(\cdot)$ on $\mathbb{R}$ with $z(t) \rightarrow(0,0)$ as $t \rightarrow-\infty$. As before, let $\varphi$ be the flow on $\mathbb{C}^{2}$ associated with this equation. (We shall keep things away from explosions!) We would like to find an injective analytic map $\Gamma$ with domain a neighbourhood of $(0,0)$ in $\mathbb{C}^{2}$ with $\Gamma(0,0)=(0,0)$ and with analytic inverse $\gamma$, such that for some simply-described flow $\theta$ (preferably our previous 'linear' flow $\mathscr{L}$ ) we have

$$
\varphi_{t} \Gamma w=\Gamma \theta_{t} w \quad(\infty<t \leqslant 0)
$$

for $w$ near $(0,0)$. We find that (generically) we can do this with $\theta=\mathscr{L}$ if and only if there is no 'resonance', that is, if and only if $\lambda_{0}$ is not an integer multiple $\lambda_{0}=\ell \lambda_{1}(\ell \geqslant 2)$ of $\lambda_{1}$. (We already know that we can arrange (2.1.2) with $\theta=\mathscr{L}$ and with $\Gamma$ a homeomorphism, for $w$ in the entire cone $K$ and all $t$ in $\mathbb{R}$, even if there is resonance.)

Algebra of power series. The idea is to take a power-series expansion

$$
z:=\Gamma w=\xi_{0} u+\xi_{1} v+\sum_{k+\ell \geqslant 2} \frac{1}{k ! \ell !}\left(A_{k \ell} u+B_{k \ell} v\right) \xi_{0}^{k} \xi_{1}^{\ell},
$$

in $\left(\xi_{0}, \xi_{1}\right)$, where $w=\xi_{0} u+\xi_{1} v\left(\xi_{i} \in \mathbb{C}\right)$. Because of (2.1.2), we require that

$$
\begin{aligned}
z(t) & :=\Gamma\left(\theta_{t} w\right) \\
& =\xi_{0}(t) u+\xi_{1}(t) v+\sum_{k+\ell \geqslant 2} \frac{1}{k ! \ell !}\left(A_{k \ell} u+B_{k \ell} v\right) \xi_{0}(t)^{k} \xi_{1}(t)^{\ell},
\end{aligned}
$$

satisfy (2.1.1) provided that $w(t)=\theta_{t} w=\xi_{0}(t) u+\xi_{1}(t) v$ satisfies some simple equation, preferably, $\dot{w}=(R+Q) w$.

We suppose that

$$
\begin{aligned}
\dot{\xi}_{0}(t) & =\lambda_{0} \xi_{0}(t)+\psi_{0}\left(\xi_{0}(t), \xi_{1}(t)\right), \\
\dot{\xi}_{1}(t) & =\lambda_{1} \xi_{1}(t)+\psi_{1}\left(\xi_{0}(t), \xi_{1}(t)\right), \\
\psi_{0}\left(\xi_{0}(t), \xi_{1}(t)\right) & =\sum_{k+\ell \geqslant 2} \frac{1}{k ! \ell !}\left(C_{k \ell} u+D_{k \ell} v\right) \xi_{0}(t)^{k} \xi_{1}(t)^{\ell},
\end{aligned}
$$


$\psi_{1}(\cdot)$ having a similar expansion and $E$ and $F$ replacing $C$ and $D$. The object is to make the series for $\psi_{0}(\cdot)$ and $\psi_{1}(\cdot)$ as simple as possible, preferably zero!

So, we substitute (2.1.5) and (2.1.4) in (2.1.1), and require that (2.1.1) holds as an identity of power series in $\left(\xi_{0}(t), \xi_{1}(t)\right)$, and $\psi_{0}(\cdot)$ and $\psi_{1}(\cdot)$ are as simple as possible. Now $\{u, v\}$ is a basis for the linear space of functions on $I$, so that a monomial $u^{r} v^{s}\left(r, s \in \mathbb{Z}_{+}\right)$is a linear combination of $u$ and $v$ with coefficients in $\mathbb{C}$. Hence, (2.1.4) implies that

$$
z(t)^{2}=\sum_{k+\ell \geqslant 2} \frac{1}{k ! \ell !}\left(G_{k \ell} u+H_{k \ell} v\right) \xi_{0}(t)^{k} \xi_{1}(t)^{\ell},
$$

where $G_{k \ell}$ and $H_{k \ell}$ are determined by values of $A_{r s}$ and $B_{r s}$ where $r+s<k+\ell$. Because of (2.1.5), we have

$$
\begin{aligned}
\frac{d}{d t}\left\{\xi_{0}(t)^{k} \xi_{1}(t)^{\ell}\right\}= & \left(k \lambda_{0}+\ell \lambda_{1}\right) \xi_{0}(t)^{k} \xi_{1}(t)^{\ell} \\
& +(\text { terms of order greater than } k+\ell) .
\end{aligned}
$$

Also,

$$
(R+Q)\left(A_{k \ell} u+B_{k \ell} v\right)=\lambda_{0} A_{k \ell} u+\lambda_{1} B_{k \ell} v .
$$

Working recursively through increasing values of $k+l$, we find that setting the coefficient of $\xi_{0}(t)^{k} \xi_{1}(t)^{\ell} u$ in (2.1.1) to zero yields the so-called homological equation for ' $u$ ' coefficients:

$$
\frac{1}{k ! \ell !}\left[C_{k \ell}+\left(k \lambda_{0}+\ell \lambda_{1}-\lambda_{0}\right) A_{k \ell}\right]=\text { (already known terms), }
$$

and we have a corresponding (2.1.6v) equation. Provided that $k \lambda_{0}+\ell \lambda_{1}-\lambda_{0} \neq 0$, we can, and $d o$, set $C_{k \ell}=0$ and solve $(2.1 .6 u)$ for $A_{k \ell}$. We note that because $\lambda_{0}>\lambda_{1}>0$, we can have $k \lambda_{0}+\ell \lambda_{1}-\lambda_{0}=0$ if and only if $k=0$ and $\lambda_{0}=\ell \lambda_{1}$, where $\ell \in \mathbb{N}$ and $\ell \geqslant 2$. Thus, at least formally, if $\lambda_{0} / \lambda_{1}$ is not an integer, then we can arrange (2.1.2) with $\psi_{0}(\cdot)$ and $\psi_{1}(\cdot)$ both identically zero, whence $\dot{w}=(R+Q) w, \theta=\mathscr{L}$, and

$$
\dot{\xi}_{0}(t)=\lambda_{0} \xi_{0}(t), \quad \dot{\xi}_{1}(t)=\lambda_{1} \xi_{1}(t) .
$$

Resonance. If we have a 'resonance' $\lambda_{0}=\ell \lambda_{1}$, the case $\ell=2$ giving the 'primary resonance', then $A_{0 \ell}$ is arbitrary and, except in freak cases, we have to choose $C_{0 \ell} \neq 0$ to make $(2.1 .6 u)$ true. Noting that resonance cannot have any effect on ' $v$ ' coefficients (because $\lambda_{0}>\lambda_{1}$ ), we see that we shall have

$$
D_{r s}=0 \text { for all }(r, s), \quad C_{r s}=0 \text { for }(r, s) \neq(0, \ell) .
$$

Thus, if $\lambda_{0}=\ell \lambda_{1}$, we have

so that

$$
\dot{\xi}_{0}(t)=\lambda_{0} \xi_{0}(t)+\frac{1}{\ell !} C_{0 \ell} \xi_{1}(t)^{\ell}, \quad \dot{\xi}_{1}(t)=\lambda_{1} \xi_{1}(t),
$$

$$
\xi_{1}(t)=\xi_{1}(0) e^{\lambda_{1} t}, \quad \xi_{0}(t)=\left\{\xi_{0}(0)+\frac{1}{\ell !} C_{0 \ell} \xi_{1}(0)^{\ell} t\right\} e^{\lambda_{0} t} .
$$

We note that on the cone $K$ the flow $\theta$ always agrees with the linear flow $\mathscr{L}$. 
Analysis of power series. The above discussion explains the 'algebra' of the formal power series. The analysis in the work of Poincare and Dulac states that $\Gamma$ may be defined on a neighbourhood of $(0,0)$ in $\mathbb{C}^{2}$ via the convergent series (2.1.3), and that (2.1.2) will hold for negative $t$ in that neighbourhood, $\xi(\cdot)$ satisfying (2.1.7) away from resonance and (2.1.9) when $\lambda_{0}=\ell \lambda_{1}$.

REMARK 2.1.1. We emphasize once more that in the generic case, $\varphi$ will have two fixed points in $\mathbb{C}^{2} \backslash E$. There will therefore never be a global isomorphism between $\varphi$ and $\theta$ as there was in the case of complex dimension 1 .

2.2. The Poincare expansion as a cumulant expansion in the case when $\lambda_{0}<2 \lambda_{1}$

Suppose in this subsection that $\lambda_{0}<2 \lambda_{1}$. Thus, $\theta$ is our linear flow $\mathscr{L}$ on $\mathbb{C}^{2}$. Then, from the probabilistic point of view which we owe principally to McKean, the series expansions (2.1.3) and (2.1.4) are exactly what statisticians call cumulant expansions:

$$
G w:=\lim _{t \rightarrow \infty} X_{t} \mathscr{L}_{-t} w \text { exists a.s. for every } w \text { in } \mathbb{C}^{2},
$$

and, for $w=\xi_{0} u+\xi_{1} v$ in a neighbourhood of $(0,0)$ in $\mathbb{C}^{2}$,

$$
\begin{aligned}
\exp \{-x \Gamma w\} & =\mathbb{E}^{x} \exp \{-G w\}=\mathbb{E}^{x} \exp \left\{-\xi_{0} M_{\infty}-\xi_{1} N_{\infty}\right\}, \\
\exp \left\{-x \varphi_{-t} \Gamma w\right\} & =\mathbb{E}^{x} \exp \left\{-G \mathscr{L}_{-t} w\right\} \quad(t \geqslant 0),
\end{aligned}
$$

all for every $x \in \mathscr{M}(I)$. For example, we can obtain $A_{02}$ and $B_{02}$ at (2.1.3) from

$$
-\operatorname{Var}^{x} N_{\infty}=x\left(A_{02} u+B_{02} v\right)
$$

where $\operatorname{Var}^{x}$ is the variance associated with $\mathbb{P}^{x}$.

The right-hand side of (2.2.2) focuses attention on where $\Gamma w$ is defined. The existence for $w$ in a neighbourhood of $(0,0)$ in $\mathbb{C}^{2}$ of the integral on the righthand side of (2.2.2) amounts to the statement that $M_{\infty}$ and $\left|N_{\infty}\right|$ have distributions with essentially exponential tails. As we shall see, Doob's Supermartingale Inequality and Gronwall's Lemma combine to yield, for some positive function $A_{0}(\cdot)$ on $\mathscr{M}(I)$,

$$
\mathbb{P}^{x}\left(M_{\infty}>y\right) \leqslant A_{0}(x) e^{-c_{0} y} \quad(y \geqslant 0), \quad \text { where } c_{0}=\frac{\lambda_{0}}{2\|u\|_{\infty}} e^{-1},
$$

with a similar (somewhat more complicated) bound for $N_{\infty}$.

EXAMPLE 2.2.1. If $r_{1}=r_{2}=2$ and we take $u=(1,1)^{\mathrm{T}}$ as the eigenvector associated with $\lambda_{0}=2$, our formula gives $c_{0}=e^{-1}$, whereas the best value of $c_{0}$ is 'immediately below 1 ' in that

$$
\mathbb{E}^{x} \exp \left(-\xi_{0} M_{\infty}\right)=\exp \left(-x \frac{\xi_{0}}{1+\xi_{0}} u\right) \quad \text { for } \Re\left(\xi_{0}\right)>-1
$$

\subsection{The second-order coefficients; and a look at $\mathscr{A}^{-}$near $(0,0)$}

(We now drop the restriction that $\lambda_{0}<2 \lambda_{1}$, but retain the Poincaré assumption that $\lambda_{1}>0$.) When $k+\ell=2$, the homological equations $(2.1 .6 u),(2.1 .6 v)$ and 
equation (2.1.8) yield

$$
\begin{aligned}
& -2 u^{2}=\frac{1}{2}\left(2 \lambda_{0}-\lambda_{0}\right) A_{20} u+\frac{1}{2}\left(2 \lambda_{0}-\lambda_{1}\right) B_{20} v, \\
& -4 u v=\left(\lambda_{0}+\lambda_{1}-\lambda_{0}\right) A_{11} u+\left(\lambda_{0}+\lambda_{1}-\lambda_{1}\right) B_{11} v, \\
& -2 v^{2}=\frac{1}{2}\left(2 \lambda_{1}-\lambda_{0}\right) A_{02} u+\frac{1}{2}\left(2 \lambda_{1}-\lambda_{1}\right) B_{02} v+\frac{1}{2} C_{02} u .
\end{aligned}
$$

Simplifying, we find that the expansions of the functions $u^{2}, u v, v^{2}$ on $I$ in terms of the basis $\{u, v\}$ determine the coefficients as follows:

$$
\begin{aligned}
& -4 u^{2}=\lambda_{0} A_{20} u+\left(2 \lambda_{0}-\lambda_{1}\right) B_{20} v, \\
& -4 u v=\lambda_{1} A_{11} u+\lambda_{0} B_{11} v, \\
& -4 v^{2}=\left[C_{02}+\left(2 \lambda_{1}-\lambda_{0}\right) A_{02}\right] u+\lambda_{1} B_{02} v,
\end{aligned}
$$

where we choose $C_{02}=0$ unless $\lambda_{0}=2 \lambda_{1}$, in which case $A_{02}$ is arbitrary. It is easily checked, using (1.3.2), that

$$
\begin{aligned}
& \text { if } \lambda_{0}<2 \lambda_{1} \text {, then } A_{02}=-4 /\left(2 \lambda_{1}-\lambda_{0}\right)<0, C_{02}=0 ; \\
& \text { if } \lambda_{0}>2 \lambda_{1} \text {, then } A_{02}=-4 /\left(2 \lambda_{1}-\lambda_{0}\right)>0, C_{02}=0 \text {; } \\
& \text { if } \lambda_{0}=2 \lambda_{1} \text {, then } C_{02}=-4<0, A_{02} \text { is arbitrary. }
\end{aligned}
$$

We now consider $\mathscr{A}^{-}$in the neighbourhood of $(0,0)$. Suppose that $k$ is close to $(0,0)$ and that

$$
\Gamma k \in \mathscr{A}^{-}, \quad k=\gamma_{0} u+\gamma_{1} v, \quad \gamma_{0}, \gamma_{1} \in \mathbb{C} .
$$

Since $\lambda_{0}>\lambda_{1}>0$, then, as $t \rightarrow \infty$,

$$
E 3 \varphi_{-t} \Gamma k=\Gamma \theta_{-t} k=e^{-\lambda_{1} t} \gamma_{1} v+o\left(e^{-\lambda_{1} t}\right)
$$

and since $v_{1}$ and $v_{2}$ have opposite signs, we must have

$$
\Re\left(\gamma_{1}\right)=0, \quad \text { so that } \gamma_{1}=\beta_{1} i\left(\beta_{1} \in \mathbb{R}\right) .
$$

Now suppose that $\lambda_{0}>2 \lambda_{1}$. Then, as $t \rightarrow \infty$,

$$
E 3 \varphi_{-t} \Gamma k=\Gamma \theta_{-t} k=e^{-\lambda_{1} t} \beta_{1} i v-e^{-2 \lambda_{1} t} \frac{1}{2} A_{02} \beta_{1}^{2} u+o\left(e^{-2 \lambda_{1} t}\right),
$$

so that, since $A_{02}>0$ and $u>0$, we must have $\beta_{1}=0$, so $\gamma_{1}=0$. Finally consider the 'resonance' case when $\lambda_{0}=2 \lambda_{1}$. Then, by (2.1.9), as $t \rightarrow \infty$,

and here,

$$
\theta_{-t} k=e^{-\lambda_{1} t} \beta_{1} i v+t e^{-2 \lambda_{1} t} \frac{1}{2} C_{02} \beta_{1}^{2} u+O\left(e^{-2 \lambda_{1} t}\right),
$$

$$
E 3 \varphi_{-t} \Gamma k=\Gamma \theta_{-t} k=\theta_{-t} k+O\left(e^{-2 \lambda_{1} t}\right) .
$$

Since $C_{02}<0$ and $u>0$, we must have $\beta_{1}=0$, so $\gamma_{1}=0$. The reader can easily check that in all cases, $\mathfrak{R}\left(\gamma_{0}\right) \geqslant 0$.

At least when $\lambda_{1}>0$, these considerations help explain why $K$ is as at (1.3.8).

\section{Martingales}

The Poincaré assumption that $\lambda_{1}>0$ is now dropped.

The fundamental idea that the linearized system (1.3.6) is associated with 'additive' martingales for $X$ while the non-linear system (1.2.1) is associated with 
'multiplicative' martingales and that 'taking logs' provides a link between the two, goes back to McKean's famous paper [8] on the FKPP equation. See also Neveu's equally significant paper [9] and Bramson's very deep papers [3] and [4].

As was mentioned in the Introduction, many of our results mimic those of Kesten and Stigum and of Athreya and Ney for discrete branching processes. See Chapter V of Athreya and Ney [2].

\subsection{Additive martingales}

We study the martingales $M$ and $N$ mentioned earlier, the definitions of which are recalled in the following theorem.

THEOREM 3.1.1. The following results hold.

(a) For $z \in \mathbb{C}^{2}$,

$$
\left\{X_{t}\left(\mathscr{L}_{-t} z\right): t \geqslant 0\right\} \text { is a martingale. }
$$

In particular, $M=\left\{M_{t}: t \geqslant 0\right\}$, where

$$
M_{t}:=X_{t}\left(\mathscr{L}_{-t} u\right)=e^{-\lambda_{0} t} X_{t}(u),
$$

and $N=\left\{N_{t}: t \geqslant 0\right\}$, where

$$
N_{t}:=X_{t}\left(\mathscr{L}_{-t} v\right)=e^{-\lambda_{1} t} X_{t}(v),
$$

are martingales.

(b) The martingale $M$ is always bounded in $L^{2}$, so that the limit $M_{\infty}$ exists almost surely and in $L^{2}$. For some positive constant $c_{0}$ and some positive function $A_{0}(\cdot)$ on $\mathscr{M}(I), \mathbb{P}^{x}\left(M_{\infty}>y\right) \leqslant A_{0}(x) e^{-c_{0} y}$ for $y \geqslant 0$.

(c) The set $\left\{M_{\infty}=0\right\}$ equals $\{\zeta<\infty\}$, almost surely.

(d) If $\lambda_{0}<2 \lambda_{1}$, then $N$ is bounded in $L^{2}, N_{\infty}$ exists almost surely, and, for some positive constant $c_{1}$ and some positive function $A_{1}(\cdot)$ on $\mathscr{M}(I)$, $\mathbb{P}^{x}\left(\left|N_{\infty}\right|>y\right) \leqslant A_{1}(x) e^{-c_{1} y}$ for $y \geqslant 0$.

(e) If $\lambda_{0} \geqslant 2 \lambda_{1}$, then $N$ is not bounded in $L^{2}$ and $N$ almost surely oscillates infinitely on the set $\{\zeta=\infty\}$ : almost surely,

$$
\zeta=\infty \text { implies that } \lim \sup N_{t}=+\infty \text { and } \liminf N_{t}=-\infty .
$$

(f) If $m$ is the right eigenmeasure in (1.3.2), then, as $t \rightarrow \infty$,

$$
e^{-\lambda_{0} t} X_{t} \rightarrow M_{\infty} m \text { almost surely. }
$$

Proof of Theorem 3.1.1. We write

$$
d Y \doteq d Z
$$

to signify that $Y-Z$ is a local martingale. Recall that

$$
d X_{t}(f) \doteq X_{t}(R+Q) f d t, \quad d\left[X_{t} f\right]=4 X_{t}\left(f^{2}\right) d t .
$$

Proofs of Parts (a) and (b). Let $z \in E$. Then, using the fact that

$$
d\left\{\mathscr{L}_{-t} z\right\}=-(R+Q) \mathscr{L}_{-t} z d t
$$

we have

$$
d\left\{X_{t} \mathscr{L}_{-t} z\right\} \doteq X_{t}(R+Q) \mathscr{L}_{-t} z d t-X_{t}(R+Q) \mathscr{L}_{-t} z d t=0 .
$$


Hence, $X_{t} \mathscr{L}_{-t} z$ is a local martingale. In particular, since $\mathscr{L}_{-t} u=e^{-\lambda_{0} t} u$, $M_{t}:=e^{-\lambda_{0} t} X_{t}(u)$ is a local martingale; and $M$ has quadratic-variation

$$
[M]_{t}=4 \int_{0}^{t} e^{-2 \lambda_{0} s} X_{s}\left(u^{2}\right) d s \stackrel{(\mathrm{b})}{\sim} 4 \int_{0}^{t} e^{-\lambda_{0} s} M_{s} d s,
$$

where $\stackrel{(b)}{\sim}$ signifies that the ratio of the two sides is bounded away from 0 and $\infty$ by deterministic constants. Now, $M$ is a non-negative local martingale, and hence a supermartingale; and hence $\mathbb{E}\left(M_{s}\right) \leqslant \mathbb{E}\left(M_{0}\right)$. Thus $\mathbb{E}[M]_{\infty}<\infty$, and (see Corollary IV.1.25 of [10]), $M$ is a true martingale bounded in $L^{2}$. But now, by the same corollary, for $z \in E, X_{t}\left(\mathscr{L}_{-t} z\right)$ is a martingale, bounded in $L^{2}$ on every finite interval.

We wish now to bound the tail of the distribution of $M_{\infty}$. Now, for $\xi>0$,

$$
V:=\exp \left\{\xi M_{t}-\frac{1}{2} \xi^{2} 4 \int_{0}^{t} e^{-2 \lambda_{0} s} X_{s}\left(u^{2}\right) d s\right\}
$$

is a non-negative local martingale, hence a supermartingale, and, by Doob's Supermartingale Inequality, we therefore have

$$
\mathbb{P}^{x}\left(\sup _{t} V_{t} \geqslant r\right) \leqslant \mathbb{E}^{x}\left(V_{0}\right) / r \quad(r>0) .
$$

Hence, for $r>\exp \{\xi x(u)\}$, with probability at least $1-\exp \{\xi x(u)\} / r$ we have

$$
\begin{aligned}
M_{t} & \leqslant 2 \xi \int_{0}^{t} e^{-2 \lambda_{0} s} X_{s}\left(u^{2}\right) d s+\xi^{-1} \log (r) \\
& \leqslant 2 \xi\|u\|_{\infty} \int_{0}^{t} e^{-\lambda_{0} s} M_{s} d s+\xi^{-1} \log (r) .
\end{aligned}
$$

Gronwall's Lemma says that for a function $g$ and a positive constant $A$,

implies

$$
M_{t} \leqslant \int_{0}^{t} g_{s} M_{s} d s+A \quad(t \geqslant 0)
$$

$$
M_{t} \leqslant A \exp \left\{\int_{0}^{t} g_{s} d s\right\} \quad(t \geqslant 0) .
$$

Hence, again for $r>\exp \{\xi x(u)\}$, we have

$$
\mathbb{P}^{x}\left\{\sup _{t} M_{t}>\frac{\log r}{\xi} \exp \left(2 \xi \frac{\|u\|_{\infty}}{\lambda_{0}}\right)\right\} \leqslant \exp \{\xi x(u)-\log r\} .
$$

The best choice of $\xi$ is $\frac{1}{2} \lambda_{0} /\|u\|_{\infty}$, and this leads to

$$
\mathbb{P}^{x}\left\{\sup _{t} M_{t}>y\right\} \leqslant A_{0}(x) e^{-c_{0} y}, \quad \text { where } c_{0}=\frac{\lambda_{0}}{2\|u\|_{\infty}} e^{-1}
$$

and for some $A_{0}(x)$, strengthening the result given at (2.2.5).

Proof of Part (c). This is an important step which anticipates much else. For our right eigenfunction $u$, define $\Gamma u$ (we shall see later that this agrees with our other uses of $\Gamma$ ) via the fact that

$$
e^{-x(\Gamma u)}=\mathbb{E}^{x} \exp \left(-M_{\infty}\right) \quad \text { for } x \in \mathscr{M}(I) .
$$


Let us see why we can do this. Suppose that $X$ is an $(R, Q, x)$-process and $Y$ is an $(R, Q, y)$-process independent of $X$. We know that $X+Y$ is an $(R, Q, x+y)$ process. With obvious notation, $M_{\infty}^{X+Y}=M_{\infty}^{X}+M_{\infty}^{Y}$, where $M_{\infty}^{X}$ and $M_{\infty}^{Y}$ are independent. Thus,

$$
\mathbb{E}^{x+y} \exp \left(-M_{\infty}\right)=\mathbb{E}^{x} \exp \left(-M_{\infty}\right) \mathbb{E}^{y} \exp \left(-M_{\infty}\right) .
$$

Since $x \mapsto \mathbb{E}^{x} \exp \left(-M_{\infty}\right)$ is clearly monotonic, it must be an exponential function. With $\{\mathscr{F}(t)\}$ as the natural augmented filtration for $X$ as before, and with the notation $\mathbb{E}_{\mathscr{F}(t)}(\cdot):=\mathbb{E}(\cdot \mid \mathscr{F}(t))$, we have, by the Markov property of $X$,

$$
\begin{aligned}
\mathbb{E}_{\mathscr{F}(t)}\left(\exp \left\{-e^{\lambda_{0} t} M_{\infty}\right\}\right) & =\mathbb{E}_{\mathscr{F}(t)}\left(\exp \left\{-\lim _{s \rightarrow \infty} e^{\lambda_{0} t} e^{-\lambda_{0}(t+s)} X_{t+s}(u)\right\}\right) \\
& =\mathbb{E}_{\mathscr{F}(t)}\left(\exp \left\{-\lim _{s \rightarrow \infty} e^{-\lambda_{0} s} X_{t+s}(u)\right\} \mid \mathscr{F}_{t}\right) \\
& =\exp \left\{-X_{t} \Gamma u\right\},
\end{aligned}
$$

almost surely. Hence,

$$
\mathbb{E} \exp \left\{-e^{\lambda_{0} t} M_{\infty}\right\}=\mathbb{E} \exp \left\{-X_{t} \Gamma u\right\} .
$$

It is easily checked from the fact that $\mathbb{E}^{y} M_{\infty}=y(u)$ for $y \in \mathscr{M}(I)$ that both components of $\Gamma u$ are positive. Since $X_{t} \rightarrow \infty$ (a.s.) on $\{\zeta=\infty\}$, the right-hand side of (3.1.7) converges, as $t \rightarrow \infty$, to $\mathbb{P}(\zeta<\infty)$. But the left-hand side converges to $\mathbb{P}\left(M_{\infty}=0\right)$. Since the condition $\zeta<\infty$ implies that $M_{\infty}=0$, we must have

$$
\left\{M_{\infty}=0\right\}=\{\zeta<\infty\}, \quad \text { almost surely; }
$$

and this is what we sought to prove.

Proof of Parts (d), (e) and (f). We now know that $N_{t}:=e^{-\lambda_{1} t} X_{t}(v)$ is a local martingale; and has quadratic-variation

$$
[N]_{t}=4 \int_{0}^{t} e^{-2 \lambda_{1} s} X_{s}\left(v^{2}\right) d s \stackrel{(\mathfrak{b})}{\sim} 4 \int_{0}^{t} e^{\left(\lambda_{0}-2 \lambda_{1}\right) s} M_{s} d s .
$$

If $\lambda_{0}<2 \lambda_{1}$, then $\mathbb{E}[N]_{\infty}<\infty$, and $N$ is a true martingale bounded in $L^{2}$. It is now easy to obtain the last part of (d) by the supermartingale method (without needing Gronwall's Lemma) applied separately to $N$ and to $(-N)$, using (3.1.8) and (3.1.4).

If $\lambda_{0} \geqslant 2 \lambda_{1}$, then $[N]_{\infty}=\infty$ a.s. on the set $\left\{M_{\infty}>0\right\}$, and hence, since $N$ is a time-transformation of Brownian motion (see [10, § V.1] or [12, § IV.34]), $N$ a.s. oscillates infinitely on $\left\{M_{\infty}>0\right\}$.

By another application of the time-transformation property, for any $p>1$, we have a.s. on $\left\{M_{\infty}>0\right\}, N_{t} /[N]^{p / 2} \rightarrow 0$. But, for $\lambda_{0} \geqslant 2 \lambda_{1}$, it is clear from (3.1.8) that a.s. on $\left\{M_{\infty}>0\right\}$,

$$
\limsup t^{-1} e^{-\left(\lambda_{0}-2 \lambda_{1}\right) t}[N]_{t}<\infty
$$

whence

$$
\limsup t^{-p / 2} e^{-p\left(\lambda_{0}-2 \lambda_{1}\right) t / 2} N_{t}<\infty
$$

and by choosing $p$ so that $\frac{1}{2} p\left(\lambda_{0}-2 \lambda_{1}\right)<\lambda_{0}-\lambda_{1}$, we see that

$$
e^{-\left(\lambda_{0}-\lambda_{1}\right) t} N_{t}=e^{-\lambda_{0} t} X_{t}(v) \rightarrow 0 \text {. }
$$


That this result also holds on $\left\{M_{\infty}=0\right\}=\{\zeta<\infty\}$ is obvious. On combining these results with the fact that $e^{-\lambda_{0} t} X_{t}(u) \rightarrow M_{\infty}$, we see that $e^{-\lambda_{0} t} X_{t} \rightarrow M_{\infty} m$, a.s., as required. (Recall that $m(u)=1$ and $m(v)=0$.)

\subsection{Multiplicative martingales; and 'taking logs'}

THeOREM 3.2.1. The following results hold.

(a) For $a \in \mathscr{A}^{-}$,

$$
W_{t}^{a}:=\exp \left\{-X_{t}\left(\varphi_{-t} a\right)\right\}
$$

defines a bounded martingale.

(b) If $z$ in $E$ is such that $\varphi_{-t} z \in E$ for $0 \leqslant t \leqslant t_{0}$, then $\exp \left\{-X_{t}\left(\varphi_{-t} z\right)\right\}$ is a bounded martingale on time-parameter set $\left[0, t_{0}\right]$.

(c) The point $e_{\infty}$ defined (see (1.2.7)) via

$$
\mathbb{P}^{x}(\zeta<\infty)=\exp \left\{-x\left(e_{\infty}\right)\right\}
$$

is the unique fixed point of $\varphi$ within $E \backslash\{(0,0)\}$.

(d) For $z \in E$ with $z \neq(0,0)$,

$$
\varphi_{t} z \rightarrow e_{\infty} \text { as } t \rightarrow \infty
$$

Proof of Theorem 3.2.1. Once Itô's formula has given us the multiplicative martingales, the rest is easy.

Proofs of Parts (a) and (b). Let $a \in \mathscr{A}^{-}$. We have

$$
\begin{aligned}
d\left\{X_{t} a(-t)\right\}= & \left\{(d B) \sigma(X)+(d t) X_{t}(R+Q)\right\} a(-t) \\
& +X_{t}\left\{-(R+Q) a(-t)-2 a(-t)^{2}\right\} d t \\
= & (d B) \sigma(X) a(-t)-2 X_{t}\left\{a(-t)^{2}\right\} d t .
\end{aligned}
$$

Hence, with $W_{t}^{a}:=\exp \left\{-X_{t} a(-t)\right\}$, Itô's formula gives,

$$
\begin{aligned}
d W_{t}^{a} & =W_{t}^{a}\left(-d\left\{X_{t} a(-t)\right\}+\frac{1}{2} 4 X\left\{a(-t)^{2}\right\} d t\right) \\
& =W_{t}^{a}(d B) \sigma(X) a(-t) \doteq 0,
\end{aligned}
$$

and $W^{a}$ is a local martingale. But $W^{a}$ is uniformly bounded in modulus by 1 , and so $W^{a}$ is a true martingale. This proves Part (a) and Part (b) follows similarly.

Proof of Part (c). We have

$$
\mathbb{P}^{x}\left(\zeta<\infty \mid \mathscr{F}_{t}\right)=\exp \left\{-X_{t}\left(e_{\infty}\right)\right\},
$$

so that $\exp \left\{-X_{t}\left(e_{\infty}\right)\right\}$ is a martingale. But, by Part (b), with $z=\varphi_{t}\left(e_{\infty}\right)$, we have

$$
\mathbb{E}^{x} \exp \left\{-X_{t}\left(e_{\infty}\right)\right\}=\mathbb{E}^{x} \exp \left\{-X_{0} \varphi_{t}\left(e_{\infty}\right)\right\}=\exp \left\{-x \varphi_{t}\left(e_{\infty}\right)\right\},
$$

and, by the martingale property just proved, the left-hand side equals $\exp \left\{-x\left(e_{\infty}\right)\right\}$. Since this is true for every $x, \varphi_{t}\left(e_{\infty}\right)=e_{\infty}$. The uniqueness assertion follows from Part (d).

Now suppose that $a$ is a fixed point of $\varphi$ within $E \backslash\{(0,0)\}$. Then, since $X_{t}^{(i)} \rightarrow \infty(i \in I)$ as $t \rightarrow \infty$, a.s. on $\{\zeta=\infty\}$, we see that $W_{\infty}^{a}$ must equal 
$I_{\{\zeta<\infty\}}$. Thus,

$$
\exp \{-x(a)\}=\mathbb{E}^{x} W_{\infty}^{a}=\exp \left\{-x\left(e_{\infty}\right)\right\}, \quad \text { for } x \in \mathscr{M}(I),
$$

and so $a=e_{\infty}$.

Proof of Part (d). Because the vector field of (1.2.1) is inward-directed on the boundary of $E$ except at $(0,0)$, it is enough to prove (d) for $z$ in the interior of $E$. But then, since $X_{t} \rightarrow(\infty, \infty)$ a.s. on $\{\zeta=\infty\}$, we have, for $z$ in the interior of $E$,

$$
\begin{aligned}
\exp \left\{-x\left(\varphi_{t} z\right)\right\} & =\mathbb{E}^{x} \exp \left\{-X_{t} z\right\} \rightarrow \mathbb{P}^{x}(\zeta<\infty) \\
& =\exp \left\{-x\left(e_{\infty}\right)\right\},
\end{aligned}
$$

and it follows that $\varphi_{t} z \rightarrow e_{\infty}$.

IMPORTANT NOTE 3.2.2. Before we consider a more substantial aspect of 'taking logs', let us consider the way in which we have just 'taken logs' to deduce from (3.2.4) that $\varphi_{t} z \rightarrow e_{\infty}$ as $t \rightarrow \infty$. We shall be using this idea repeatedly, and sometimes to discuss uniformity of convergence. Because of the potential multi-valued nature of the logarithm function, it may be helpful to note the following idea which can be used at all relevant points in the paper. For any $t_{0}>0$, we can choose $x$ in $\mathscr{M}(I)$ so small that $\mathbb{P}^{x}(\zeta \leqslant t)>\frac{2}{3}$ for $t \geqslant t_{0}$. Then, for $t \geqslant t_{0}$,

$$
\mathbb{E}^{x} \exp \left(-X_{t} z\right)=\mathbb{E}^{x}\left\{\exp \left(-X_{t} z\right) ; \zeta \leqslant t\right\}+\mathbb{E}^{x}\left\{\exp \left(-X_{t} z\right) ; \zeta>t\right\},
$$

and it follows easily that (for $z \in E$ )

$$
\left|\mathbb{E}^{x} \exp \left(-X_{t} z\right)-1\right|<\frac{2}{3} \text {. }
$$

We are therefore obviously safe from the problem of multiple values if we restrict $x$ to a small neighbourhood of the origin.

The serious business of 'taking logs' is to relate multiplicative martingales to additive ones. Part (a) of the following theorem is a crucial step.

THEOREM 3.2.3. The following statements hold.

(a) Let $a \in \mathscr{A}^{-}$. Then

$$
g(a):=\lim X_{t}\left(\varphi_{-t} a\right) \text { exists almost surely and in } L^{1},
$$

whence $\varphi_{-t} a \rightarrow(0,0)$ as $t \rightarrow \infty$.

(b) Again, let $a \in \mathscr{A}^{-}$. Then, for some unique $k \in K$,

$$
g(a)=G(k), \quad \text { almost surely, }
$$

where

$$
G(k):=\lim X_{t}\left(\mathscr{L}_{-t} k\right) \text { exists almost surely and in } L^{2} .
$$

With the notation of (1.3.8),

$$
\begin{aligned}
& \text { if } \lambda_{0}<2 \lambda_{1} \text {, then } G(k):=\left(\alpha_{0}+\beta_{0} i\right) M_{\infty}+\beta_{1} i N_{\infty}, \\
& \text { if } \lambda_{0} \geqslant 2 \lambda_{1} \text {, then } G(k):=\left(\alpha_{0}+\beta_{0} i\right) M_{\infty} .
\end{aligned}
$$


(c) The inverse bijections

$$
\Gamma: K \rightarrow \mathscr{A}^{-}, \quad \gamma: \mathscr{A}^{-} \rightarrow K,
$$

are defined probabilistically as follows.

For $k \in K$, we may define $\Gamma k=\Gamma(k)$ via

$$
\exp \{-x(\Gamma k)\}=\mathbb{E}^{x} \exp \{-G(k)\}, \quad \text { for } x \in \mathscr{M}(I) ;
$$

and then $a=\Gamma(k)$ is the unique $a \in \mathscr{A}^{-}$such that (3.2.6) holds.

For $a \in \mathscr{A}^{-}, \gamma a=\gamma(a)$ is given by the fact that

$$
x(\gamma a)=\mathbb{E}^{x} g(a), \quad \text { for } x \in \mathscr{M}(I),
$$

and then $k=\gamma(a)$ is the unique $k \in K$ such that (3.2.6) holds.

Proof of Theorem 3.2.3. Here, things work out very neatly.

Proof of Part (a). Let $a \in \mathscr{A}^{-}$, and write $a(-t)$ for $\varphi_{-t} a$. We know that the continuous martingale $W_{t}^{a}$ converges. Hence, the sum of the quadratic variations over $[0, \infty)$ of its real and imaginary parts converges. But we see from (3.2.3) that this sum is

$$
\int_{0}^{\infty}\left|W_{t}^{a}\right|^{2} X_{t}\left\{|a(-t)|^{2}\right\} d t
$$

regarding $|a(-t)|^{2}$ as $\left(\left|a(-t)_{1}\right|^{2},\left|a(-t)_{2}\right|^{2}\right)^{\mathrm{T}} \in \mathbb{R}^{2}$. Since $a \neq e_{\infty}$, we can choose $x \in(0, \infty)^{2}$ with

$$
\exp \{-x(a)\} \neq \exp \left\{-x\left(e_{\infty}\right)\right\} .
$$

If it were the case that $W_{\infty}^{a}=0, \mathbb{P}^{x}$ almost surely on $\{\zeta=\infty\}$, then we would have

$$
\exp \{-x(a)\}=\mathbb{E}^{x}\left(W_{\infty}^{a}\right)=\mathbb{P}^{x}(\zeta<\infty)=\exp \left\{-x\left(e_{\infty}\right)\right\}
$$

a contradiction. Hence there is a subset of $\{\zeta=\infty\}$ of positive measure on which $W_{\infty}^{a} \neq 0$. Since $X_{t} \sim e^{\lambda_{0} t} M_{\infty} m$, it follows that

$$
\int_{0}^{\infty} e^{\lambda_{0} t}\|a(-t)\|^{2} d t<\infty
$$

Since $u$ and $v$ span $\mathbb{R}^{2}$ and $M$ and $N$ are martingales, it is clear that $\mathbb{E}^{x} X_{t}^{(i)}=O\left(e^{\lambda_{0} t}\right)$ for $i \in I$. Hence, from inequality (3.2.10),

$$
\int_{0}^{\infty} \mathbb{E} X_{t}\left\{|a(-t)|^{2}\right\} d t<\infty, \quad \int_{0}^{\infty} \mathbb{E}\|\sigma(X) a(-t)\|^{2} d t<\infty .
$$

But now, on looking at the semimartingale expression (3.2.2) for $X$, we see that

$$
g(a):=\lim X_{t}\left(\varphi_{-t} a\right) \text { exists a.s. and in } L^{1},
$$

as required. Because $X_{t} \sim e^{\lambda_{0} t} M_{\infty} m$, the existence of $g(a)$ implies that $\varphi_{-t}(a) \rightarrow(0,0)$ as $t \rightarrow \infty$.

Proof of Part (b). Additive Property 1.2.1 implies that for each $a \in \mathscr{A}^{-}$, there exists

$$
\gamma(a)=\gamma_{0}(a) u+\gamma_{1}(a) v \in E \quad\left(\gamma_{0}, \gamma_{1} \in \mathbb{C}\right)
$$


such that

$$
x(\gamma(a))=\mathbb{E}^{x} g(a) \quad(x \in \mathscr{M}(I)) .
$$

We now make this the fundamental definition of $\gamma$. The Markov property of $X$ now implies that

$$
\begin{aligned}
\mathbb{E}\left\{g(a) \mid \mathscr{F}_{t}\right\} & =\lim _{s \rightarrow \infty} \mathbb{E}\left\{X_{t+s} \varphi_{-(t+s)} a \mid \mathscr{F}_{t}\right\} \\
& =\lim _{s \rightarrow \infty} \mathbb{E}^{X_{t}}\left\{X_{s} \varphi_{-s} \varphi_{-t} a\right\}=X_{t} \gamma\left(\varphi_{-t} a\right),
\end{aligned}
$$

whence

and

$$
x(\gamma a)=\mathbb{E}^{x} X_{t} \gamma\left(\varphi_{-t} a\right)=x\left\{\mathscr{L}_{t} \gamma\left(\varphi_{-t} a\right)\right\},
$$

We therefore have

$$
\gamma(a)=\mathscr{L}_{t} \gamma\left(\varphi_{-t} a\right)
$$

$$
E 3 \gamma\left(\varphi_{-t} a\right)=\mathscr{L}_{-t} \gamma(a)=\gamma_{0}(a) e^{-\lambda_{0} t} u+\gamma_{1}(a) e^{-\lambda_{1} t} v ;
$$

and since $\lambda_{0}>\lambda_{1}$ and $v_{1}$ and $v_{2}$ have opposite signs, we see that $\gamma_{1}(a)$ must be purely imaginary. We clearly must have $\mathfrak{R} \gamma_{0}(a) \geqslant 0$.

We have shown that

$$
\mathbb{E}^{x}\left\{g(a) \mid \mathscr{F}_{t}\right\}=\gamma_{0}(a) M_{t}+\gamma_{1}(a) N_{t} .
$$

But, by a well-known theorem of Lévy (see, for example, Theorems II.50.3 and II.69.5 of [11]), the left-hand side converges to $g(a)$.

Recall that $K$ is defined as follows:

$$
\begin{aligned}
& \text { if } \lambda_{0}<2 \lambda_{1} \text {, then } K:=\left\{\left(\alpha_{0}+\beta_{0} i\right) u+\beta_{1} i v: \alpha_{0}, \beta_{0}, \beta_{1} \in \mathbb{R}, \alpha_{0} \geqslant 0\right\}, \\
& \text { if } \lambda_{0} \geqslant 2 \lambda_{1} \text {, then } K:=\left\{\left(\alpha_{0}+\beta_{0} i\right) u: \alpha_{0}, \beta_{0} \in \mathbb{R}, \alpha_{0} \geqslant 0\right\},
\end{aligned}
$$

and that $G(k)$ is defined via the statements:

$$
\begin{aligned}
& \text { if } \lambda_{0}<2 \lambda_{1} \text {, then } G(k):=\left(\alpha_{0}+\beta_{0} i\right) M_{\infty}+\beta_{1} i N_{\infty}, \\
& \text { if } \lambda_{0} \geqslant 2 \lambda_{1} \text {, then } G(k):=\left(\alpha_{0}+\beta_{0} i\right) M_{\infty} .
\end{aligned}
$$

If $\lambda_{0}<2 \lambda_{1}$, then $M_{\infty}$ and $N_{\infty}$ exist, and clearly

$$
g(a)=\gamma_{0}(a) M_{\infty}+\gamma_{1}(a) N_{\infty},
$$

and $g(a)=G(k)$, where $\alpha_{0}+\beta_{0} i=\gamma_{0}(a)$ and $\beta_{1} i=\gamma_{1}(a)$. If $\lambda_{0} \geqslant 2 \lambda_{1}$, then $N_{\infty}$ fails to exist, whence $\gamma_{1}(a)=0$ and $g(a)=G(k)$, where $\alpha_{0}+\beta_{0} i=\gamma_{0}(a)$. We now have

$$
x(\gamma a)=\mathbb{E}^{x} g(a)=\mathbb{E}^{x} G(k)=x(k),
$$

the last inequality by the martingale property of $M$ (and of $N$ if $\lambda_{0}<2 \lambda_{1}$ ). Hence,

and since

$$
\gamma a=k \in K, \quad \gamma: \mathscr{A}^{-} \rightarrow K,
$$

$$
\exp \{-x(a)\}=\mathbb{E}^{x} W_{0}^{a}=\mathbb{E}^{x} W_{\infty}^{a}=\mathbb{E}^{x} \exp \{-g(a)\}=\mathbb{E}^{x} \exp \{-G(k)\},
$$

we see that

$$
\gamma: \mathscr{A}^{-} \rightarrow K \text { is injective. }
$$


Proof of Part (c). Suppose first, and until further notice, that

$$
\lambda_{0}<2 \lambda_{1},
$$

so that $M_{\infty}$ and $N_{\infty}$ exist. For $k=\left(\alpha_{0}+\beta_{0} i\right) u+\beta_{1} i v \in K$, we now define $\Gamma k=\Gamma(k)$ via

$$
\exp \{-x(\Gamma k)\}=\mathbb{E}^{x} \exp \{-G(k)\}, \quad \text { for } x \in \mathscr{M}(I) .
$$

(Compare the discussion following (3.1.6).) Then, with $\gamma_{0}:=\alpha_{0}+\beta_{0} i$ and $\gamma_{1}:=\beta_{1} i$,

$$
\begin{aligned}
& \mathbb{E}^{x}\left(\exp \left\{-\gamma_{0} M_{\infty}-\gamma_{1} N_{\infty}\right\} \mid \widetilde{\mathscr{F}}_{t}\right) \\
& \quad=\lim _{s \rightarrow \infty} \mathbb{E}^{x}\left(\exp \left\{-\gamma_{0} e^{-\lambda_{0}(t+s)} X_{t+s}(u)-\gamma_{1} e^{-\lambda_{1}(t+s)} X_{t+s}(v)\right\} \mid \widetilde{\mathscr{F}}_{t}\right) \\
& \quad=\exp \left\{-X_{t} \Gamma\left(\mathscr{L}_{-t} k\right)\right\}
\end{aligned}
$$

and, using Part (a) of Theorem 3.2.1 at one stage, we have

whence

$$
\exp \{-x(\Gamma k)\}=\mathbb{E} \exp \left\{-X_{t} \Gamma\left(\mathscr{L}_{-t} k\right)\right\}=\exp \left\{-x \varphi_{t} \Gamma\left(\mathscr{L}_{-t} k\right)\right\},
$$

$$
\Gamma k=\varphi_{t} \Gamma \mathscr{L}_{-t} k \in \varphi_{t} E .
$$

Hence $\Gamma: K \rightarrow \mathscr{A}$. Since $\varphi_{-t} \Gamma k=\Gamma \mathscr{L}_{-t} k \rightarrow 0$ as $t \rightarrow \infty$, we have $\Gamma k \neq e_{\infty}$.

We now need a 'large $t$ ' expansion for $\Gamma \mathscr{L}_{-t} k$. We have

$$
\begin{aligned}
\exp \left\{-x\left(\Gamma \mathscr{L}_{-t} k\right)\right\} & =\mathbb{E}^{x} \exp \left\{-\gamma_{0} e^{-\lambda_{0} t} M_{\infty}-\gamma_{1} e^{-\lambda_{1} t} N_{\infty}\right\} \\
& =1-\gamma_{0} e^{-\lambda_{0} t} x(u)-\gamma_{1} e^{-\lambda_{1} t} x(v)+O\left(e^{-2 \lambda_{1} t}\right) .
\end{aligned}
$$

Here, we have used the facts that $M_{\infty}$ and $N_{\infty}$ are in $L^{2}$ and that, for $\Re(z)>0$,

$$
\begin{aligned}
\left|e^{-z}-1\right| & =\left|\int_{0}^{1}(-z) e^{-r z} d r\right|<|z|, \\
\left|e^{-z}-1+z\right| & =\left|\int_{0}^{1}(-z)\left\{e^{-r z}-1\right\} d r\right| \leqslant\left|\int_{0}^{1}\right| z|(r|z|) d r| \leqslant \frac{1}{2}|z|^{2} .
\end{aligned}
$$

From (3.2.13) and (3.2.14), we have

$$
\varphi_{-t} \Gamma k=\Gamma \mathscr{L}_{-t} k=\mathscr{L}_{-t} k+O\left(e^{-2 \lambda_{1} t}\right) .
$$

But now, almost surely,

$$
X_{t}\left(\varphi_{-t} \Gamma k\right)=X_{t}\left(\mathscr{L}_{-t} k\right)+O\left(e^{-\left(2 \lambda_{1}-\lambda_{0}\right) t}\right) \rightarrow G(k) .
$$

Hence, $g(\Gamma k)=G(k)$ and, for $x \in \mathscr{M}(I)$,

$$
\begin{aligned}
x(\gamma \Gamma k) & =\mathbb{E}^{x} g(\Gamma k)=\mathbb{E}^{x} G(k) \\
& =\gamma_{0} \mathbb{E}^{x} M_{\infty}+\gamma_{1} \mathbb{E}^{x} N_{\infty} \\
& =\gamma_{0} \mathbb{E}^{x} M_{0}+\gamma_{1} \mathbb{E}^{x} N_{0}=x(k),
\end{aligned}
$$

so that

$$
\gamma \Gamma k=k, \quad \text { for } k \in K,
$$

and $\Gamma$ is injective. Since $\gamma$ is also injective (proved at (3.2.12)), $\Gamma$ and $\gamma$ are bijections. 
When $\lambda_{0} \geqslant 2 \lambda_{1}$, the proofs that $\gamma$ and $\Gamma$ are bijections with

$$
a=\Gamma k \Longleftrightarrow \gamma a=k \Longleftrightarrow g(a)=G(k) \text { almost surely, }
$$

etc., are more-or-less the same; and in those cases, we have

$$
\varphi_{-t} \Gamma k=\Gamma \mathscr{L}_{-t} k=\mathscr{L}_{-t} k+O\left(e^{-2 \lambda_{0} t}\right) .
$$

This completes the proof of Theorem 3.2.3.

Proof of result in Remark 1.3.4. Let $a \in \mathscr{A}^{-} \cap \mathbb{R}^{2}$. Then by (3.2.5), $g(a)$ is real, whence, from (3.2.9), $k:=\gamma a$ is real. Hence $k=e^{\lambda_{0} t} u=\mathscr{L}_{t} u$ for some $t \in[-\infty, \infty)$. Hence, if we write $w=\Gamma u$, then

$$
\mathscr{A} \cap \mathbb{R}^{2}=\left\{\varphi_{t} w:-\infty \leqslant t \leqslant \infty\right\},
$$

with, of course, $\varphi_{-\infty} w=0$ and $\varphi_{\infty} w=e_{\infty}$.

Discussion of equation (1.1.1). Suppose that $r_{1}=r_{2}=2$ and that $q_{1}+q_{2} \geqslant 1$. Then

$$
\lambda_{0}=2, \quad \lambda_{1}=2-\left(q_{1}+q_{2}\right), \quad u=\left(\begin{array}{l}
1 \\
1
\end{array}\right), \quad v=\left(\begin{array}{r}
-1 \\
1
\end{array}\right), \quad m=\left(\frac{1}{2}, \frac{1}{2}\right) .
$$

We have $\lambda_{0} \geqslant 2 \lambda_{1}$. Let $a \in \mathscr{A}^{-}$. Then, from (3.2.8), we have, for $x \in \mathscr{M}(I)$,

$$
\exp \{-x(a)\}=\mathbb{E}^{x} \exp \left\{-\left(\alpha_{0}+\beta_{0} i\right) M_{\infty}\right\}
$$

for some $\alpha_{0} \in[0, \infty)$ and $\beta_{0} \in \mathbb{R}$. However, because $(R+Q) u=2 u$ and $u^{2}=u$, the process $\left\{X_{t} u\right\}$ is an autonomous Markov process; see equations (1.2.4) and (1.2.5). Hence, whenever $x$ and $y$ in $\mathscr{M}(I)$ are such that $x_{1}+x_{2}=y_{1}+y_{2}$, we have

$$
\exp \{-x(a)\}=\exp \{-y(a)\} \text {. }
$$

The only way in which this can happen is that $a_{1}=a_{2}$.

\section{Probability and analysis}

\subsection{Proof of Theorem 1.3.1}

The fact that $\mathscr{A}$ consists of $(0,0), e_{\infty}$ and the tracks of heteroclinic orbits within $E$ going from $(0,0)$ to $e_{\infty}$ follows from Parts (c) and (d) of Theorem 3.2.1 and Part (a) of Theorem 3.2.3.

We wish to apply the Riemann-Lebesgue Lemma to deduce Theorem 1.3.1 with $\varphi_{t}\left(\infty_{E}\right)$ as the $e_{t}$ in Probability Properties 1.2.2. The operator (infinitesimal generator) $\mathscr{G}$ associated with $X$, namely,

$$
\mathscr{G}:=2 x^{(1)} D_{1}^{2}+2 x^{(2)} D_{2}^{2}+x(R+Q)\left(\begin{array}{c}
D_{1} \\
D_{2}
\end{array}\right), \quad \text { where } D_{i}:=\frac{\partial}{\partial x^{(i)}},
$$

has singular behaviour on the axes; so some care is necessary.

Near a point $\left(0, x^{(2)}\right)$ where $x^{(2)}>0$, the $X^{(1)}$ component of $X$ behaves like the squared Bessel process of 'dimension' $x^{(2)} q_{2}$ in a sense 'quantified' in the next paragraph. Section XI.1 of [10] and $\S 48$ and again (the Yamada-Ikeda-Watanabe Comparison) Theorem V.43.1 of [12] provide what we need here. Now, if started away from 0 , a squared Bessel process of dimension $d>0$ can hit 0 if and only if 
$d<2$; but the process almost surely spends zero time at 0 . Our process $X$, started at a point $x$ away from $(0,0)$, therefore has zero probability of hitting $\{0\} \times\left[2 q_{2}^{-1}, \infty\right)$ and positive probability of hitting any open subinterval, named in advance, of $\{0\} \times\left(0,2 q_{2}^{-1}\right)$. However, unless it hits $(0,0), X$ almost surely spends zero time on the axes; and hence, by Fubini's Theorem, for almost every fixed $t>0, X_{t}$ is almost surely either at $(0,0)$ or in $(0, \infty)^{2}$. Thus, there is a Lebesgue-null Borel subset $N_{x}$ of $[0, \infty)$ such that for $t \notin N_{x}$,

$$
\mathbb{P}^{x}[X(t) \in(\{0\} \times(0, \infty)) \cup((0, \infty) \times\{0\})]=1 .
$$

We do not need the intuitively obvious (but not-easy-to-prove) fact that $N_{x}$ is empty.

Indeed, the only fact from the previous paragraph which we actually need for this paper is that $X$ almost surely spends zero time in $\{0\} \times(0, \infty)$ (whence, by symmetry, it almost surely spends zero time in $(0, \infty) \times\{0\})$. Here are the details of how to prove this by the Comparison Theorem. (The other assertions in the previous paragraph are proved similarly.) Fix the starting point $x$ for the moment. Suppose that there is a positive probability that $X$ spends positive time in $\{0\} \times(0, \infty)$. Then, by the Monotone-Convergence Theorem, for some $\varepsilon^{(2)}>0$, $X$ spends positive time in $\{0\} \times\left[2 \varepsilon^{(2)}, \infty\right)$. Let $\varepsilon^{(1)}>0$ be such that $\delta^{(1)}=\varepsilon^{(1)}\left(r_{1}-q_{1}\right)+\varepsilon^{(2)}>0$. Our supposition implies that there must exist rational times $t_{1}$ and $t_{2}$ with $t_{2}>t_{1}$ such that with positive probability, during the time-interval $\left[t_{1}, t_{2}\right]$,

$X$ is restricted within $\left[0, \varepsilon^{(1)}\right] \times\left[\varepsilon^{(2)}, \infty\right)$, and

$X^{(1)}$ spends positive time at 0 .

Now drop the supposition. We have learnt that to prove the desired result, it is enough (recall the Markov property!) to prove that if $\varepsilon^{(2)}>0, \varepsilon^{(1)}>0$ and $\delta^{(1)}=\varepsilon^{(1)}\left(r_{1}-q_{1}\right)+\varepsilon^{(2)}>0$, and if $x \in\left[0, \varepsilon^{(1)}\right] \times\left[\varepsilon^{(2)}, \infty\right)$ and $T$ is the stopping time

$$
T:=\inf \left\{t: X_{t} \notin\left[0, \varepsilon^{(1)}\right] \times\left[\varepsilon^{(2)}, \infty\right)\right\},
$$

then $X^{(1)}$ almost surely spends zero time at 0 during the time-interval $[0, T]$. Now,

$$
d X^{(1)}=2 \sqrt{X^{(1)}} d B^{(1)}+\left\{X^{(1)}\left(r_{1}-q_{1}\right)+X^{(2)} q_{2}\right\} d t .
$$

Define the 1-dimensional process $Y$ (a squared-Bessel process of 'dimension' $\delta^{(1)}$ ) to be the pathwise unique solution (see Definition V.9.4 and $\S \mathrm{V} .48$ of [12]) of the exact equation

$$
d Y=2 \sqrt{Y} d B^{(1)}+\delta^{(1)} d t .
$$

On applying the Comparison Theorem up to time $T$ (just replace $t$ by $t \wedge T$ in the le Gall proof of Theorem V.43.1 of [12]), we see that $X_{t}^{(1)} \geqslant Y_{t}$ on $[0, T]$, whence $X^{(1)}$ almost surely spends zero time at 0 during $[0, T]$ because the same is true for $Y$. In applying Theorem V.43.1 of [12], take $X^{1}, X^{2}, \sigma(x), B, b_{1}(x), b_{2}(x)$ there to be our $X^{(1)}, Y, 2 \sqrt{x}, B^{(1)}, \delta^{(1)}, \delta^{(1)}$ respectively.

Again let $X$ start at the point $x$. If $f$ on $[0, \infty) \times[0, \infty)^{2}$ is smooth and has compact support lying within the open region $(0, \infty) \times(0, \infty)^{2}$, then

$$
f\left(t, X_{t}\right)-\int_{0}^{t}\left(\frac{\partial}{\partial t}+\mathscr{G}\right) f\left(s, X_{s}\right) d s
$$

is a martingale, whence the formal density, a Schwartz distribution, $(t, y) \mapsto p(t, y)$ 
of $X_{t}$ on $(0, \infty) \times(0, \infty)^{2}$ under $\mathbb{P}^{x}$ satisfies

$$
\left(-\frac{\partial}{\partial t}+\mathscr{G}^{*}\right) p=0
$$

Hörmander's Hypoellipticity Theorem (or, indeed, Weyl's Lemma) guarantees that $p$ is a true smooth function. See Theorem 22.2.1 of Hörmander [7].

Since for $t \notin N_{x}, p(t, \cdot)$ is an $L^{1}$ function on $(0, \infty)^{2}$, the Riemann-Lebesgue Lemma now implies that if $t \notin N_{x}$, then as $z \rightarrow \infty$ within $E$,

$$
\begin{aligned}
\exp \left\{-x\left(\varphi_{t} z\right)\right\} & =\mathbb{E}^{x} \exp \left\{-X_{t}(z)\right\} \\
& =\int_{(0, \infty)^{2}} e^{-y(z)} p(t, y) d y+\mathbb{P}^{x}(\zeta \leqslant t) \\
& \rightarrow \mathbb{P}^{x}(\zeta \leqslant t)=\exp \left\{-x\left(e_{t}\right)\right\} .
\end{aligned}
$$

That $\varphi_{t} z \rightarrow e_{t}$ as $z \rightarrow \infty$ within $E$ for every $t>0$ is now easily deduced: if we have a Lebesgue-null subset $N$ of $[0, \infty)$, then we can write any $t>0$ as $t_{1}+t_{2}$ with $t_{1}$ and $t_{2}$ in $[0, \infty) \backslash N$.

\subsection{Proof of Theorem 1.3.2}

The fact that $\varphi_{t} \mathscr{L}_{-t} k \rightarrow \Gamma k$ for $k \in K$ is proved and extended in our proof of Theorem 1.5.1 which is given below. If $a \in \mathscr{A}^{-}$, then $a=\Gamma k$ for some $k \in K$, and

$$
\mathscr{L}_{t} \varphi_{-t} a=\mathscr{L}_{t} \varphi_{-t} \Gamma k=\mathscr{L}_{t} \Gamma \mathscr{L}_{-t} k,
$$

which expression converges to $k=\gamma a$ because of the estimates (3.2.15) and (3.2.17). These two estimates feature in Theorem 1.3.2.

We now turn to the proof that the map $\Gamma$ extends to a homeomorphism of $\bar{K}$ to $\mathscr{A}$. The map

$$
k \mapsto \mathbb{E}^{x} \exp \{-G(k)\} \quad(=\exp \{-x(\Gamma k)\})
$$

is continuous on $K$ by the Dominated-Convergence Theorem. We must now prove that

$$
\text { if } k \in K \text { and }\|k\| \rightarrow \infty \text {, then } \Gamma k \rightarrow e_{\infty} .
$$

Note. Suppose we knew that, apart from an atom at $0, M_{\infty}$ has an absolutely continuous distribution on $\mathbb{R} \backslash\{0\}$, and that the same is true for $N_{\infty}$ when $N_{\infty}$ exists. Then we could deduce (4.2.1) from the Riemann-Lebesgue Lemma. However, as we have just seen(!), it is not that easy to establish absolute continuity; and we therefore adopt a different method.

Proof of (4.2.1). We have already used the fact that, because the vector field of $\varphi$ points inwards at all boundary points of $E$ other than $(0,0)$, we have

for $a \in \mathscr{A}$ and $j \in\{1,2\}, \Re\left(a_{j}\right)=0$ implies that $a=(0,0)$.

Let $\widehat{K}:=\{\widehat{k} \in K:\|\widehat{k}\|=1\}$. Then it follows from (4.2.2), the compactness of $\widehat{K}$ and injective character of $\Gamma$ on $K$ that

$$
\eta:=\inf \left\{\Re\left(\Gamma(\widehat{k})_{j}\right): \widehat{k} \in \widehat{K}, j \in\{1,2\}\right\}>0 .
$$

Fix $x \in \mathscr{M}(I)$. For $\widehat{k} \in \widehat{K}$,

$$
\exp \left\{-x\left(\Gamma \mathscr{L}_{t} \widehat{k}\right)\right\}=\exp \left\{-x\left(\varphi_{t} \Gamma \widehat{k}\right)\right\}=\mathbb{E}^{x} \exp \left\{-X_{t}(\Gamma \widehat{k})\right\},
$$


so that

$$
\begin{aligned}
\exp \left\{-x\left(\Gamma \mathscr{L}_{t} \widehat{k}\right)\right\}= & \mathbb{E}^{x}\left(\exp \left\{-X_{t}(\Gamma \widehat{k})\right\} ; \zeta \leqslant t\right) \\
& +\mathbb{E}^{x}\left(\exp \left\{-X_{t}(\Gamma \widehat{k})\right\} ; t<\zeta<\infty\right) \\
& +\mathbb{E}^{x}\left(\exp \left\{-X_{t}(\Gamma \widehat{k})\right\} ; \zeta=\infty\right) .
\end{aligned}
$$

Of the three terms on the right-hand side, the first equals $\mathbb{P}^{x}(\zeta \leqslant t)$; the second is dominated in modulus uniformly over $\widehat{k} \in \widehat{K}$ by $\mathbb{P}^{x}(t<\zeta<\infty)$; and the third is dominated in modulus uniformly over $\widehat{k} \in \widehat{K}$ by $\mathbb{E}^{x}\left(\exp \left\{-\eta X_{t}(1)\right\}, \quad \zeta=\infty\right)$. Hence, for $x \in \mathscr{M}(I)$,

$$
\exp \left\{-x\left(\Gamma \mathscr{L}_{t} \widehat{k}\right)\right\} \rightarrow \mathbb{P}^{x}(\zeta<\infty)=\exp \left\{-x\left(e_{\infty}\right)\right\} \quad \text { as } t \rightarrow \infty,
$$

uniformly over $\widehat{k} \in \widehat{K}$. Result (4.2.1) now follows because any $k$ in $K$ may be written as $\mathscr{L}_{t} \widehat{k}$ where $\widehat{k} \in \widehat{K}$ and

$$
\|k\|=\left\|\mathscr{L}_{t} \widehat{k}\right\| \leqslant e^{\lambda_{0} t}\|\widehat{k}\|=e^{\lambda_{0} t},
$$

so that $t \geqslant \lambda_{0}^{-1} \log \|k\|$. The proof of (4.2.1) is complete.

Since $\Gamma$ is a continuous bijection on the compact set $\bar{K}$, it is a homeomorphism of $\bar{K}$ onto $\mathscr{A}$.

If $\lambda_{0}<2 \lambda_{1}$, then $\bar{K}$, and hence also $\mathscr{A}$, are homeomorphic to

$$
\left\{\left(\alpha_{0}, \beta_{0}, \beta_{1}\right) \in \mathbb{R}^{3}: \alpha_{0} \geqslant 0\right\} \cup\{\infty\},
$$

which (by inversion) is homeomorphic to the unit ball in $\mathbb{R}^{3}$. If $\lambda_{0} \geqslant 2 \lambda_{1}$, then $\bar{K}$ and $\mathscr{A}$ are homeomorphic to the unit ball in $\mathbb{R}^{2}$.

The remaining parts of Theorem 1.3.2 are left to the reader.

Note. All parts of Theorem 1.4.1 were proved in $\S 3$.

\subsection{Asymptotic Gaussian behaviour, and proof of Theorem 1.5.1}

At least formally, the convergences in Theorem 1.5.1 follow (when $\lambda_{1}>0$ ) from the Poincaré idea, $C$ being $A_{02}$ and $C^{*}$ being $-C_{02}$. The probabilistic argument is rigorous and global.

Proof of Theorem 1.5.1. Throughout the proof, we make repeated use of the fact that if $\xi$ and $\eta$ are complex numbers with $\Re(\xi) \geqslant 0$ and $\Re(\eta) \geqslant 0$, then

$$
\left|e^{-\xi}-e^{-\eta}\right| \leqslant|\xi-\eta| \text {. }
$$

It should be noted that the definitions in (a), (b) and (c) of Theorem 1.5.1 do guarantee that (1.5.2), (1.5.3) and (1.5.4) hold.

Suppose first that $\lambda_{0}<2 \lambda_{1}$. As usual, this is the easiest case. With the definitions in (a) of Theorem 1.5.1, we have

$$
\begin{aligned}
\exp \left\{-x\left(\varphi_{t} s_{-t} z\right)\right\} & =\mathbb{E}^{x} \exp \left\{-X_{t}\left(s_{-t} z\right)\right\} \\
& =\mathbb{E}^{x} \exp \left\{-\left(\alpha_{0}+\beta_{0} i\right) M_{t}-\alpha_{1} e^{-\left(\lambda_{0}-\lambda_{1}\right) t} N_{t}-\beta_{1} i N_{t}\right\} .
\end{aligned}
$$

But $M_{t} \rightarrow M_{\infty}$ and $N_{t} \rightarrow N_{\infty}$ (a.s.), and $e^{-\left(\lambda_{0}-\lambda_{1}\right) t} N_{t} \rightarrow 0$ in $L^{1}$. Hence, by the 
Dominated-Convergence Theorem and (4.3.1), we have

$$
\begin{aligned}
\exp \left\{-x\left(\varphi_{t} s_{-t} z\right)\right\} & \rightarrow \mathbb{E}^{x} \exp \left\{-\left(\alpha_{0}+\beta_{0} i\right) M_{\infty}-\beta_{1} i N_{\infty}\right\} \\
& =\exp \{-x(\Gamma \Pi z)\} .
\end{aligned}
$$

Now suppose that $\lambda_{0}>2 \lambda_{1}$, let $\mu:=\frac{1}{2}\left(\lambda_{0}-2 \lambda_{1}\right)>0$, and define $s_{-t}$ and $\Pi$ as in (b) of Theorem 1.5.1. Then

$$
d N_{t}=e^{\mu t} H_{t} d b_{t}, \quad \text { with } b \text { a Brownian motion relative to }\left\{\tilde{\mathscr{F}}_{t}\right\},
$$

where $H_{t} \geqslant 0$ and

$$
H_{t}^{2}=e^{-\lambda_{0} t} 4 X_{t}\left(v^{2}\right)=e^{-\lambda_{0} t} 4 X_{t}(u+\rho v)
$$

and it is almost immediate that $\mathbb{E}^{x}\left(H_{t}^{2}\right) \rightarrow 4 \mathbb{E}^{x} M_{\infty}$. However, since $e^{-\lambda_{0} t} X_{t} \rightarrow M_{\infty} m$ (a.s.), we also have $H_{t}^{2} \rightarrow 4 M_{\infty} m(u+\rho v)=4 M_{\infty}$, a.s. Hence, $H_{t} \rightarrow 2 \sqrt{M_{\infty}}$ in $L^{2}$. For $t \geqslant t_{0}$, we have

$$
e^{-\mu t} N_{t}=e^{-\mu t} N_{t_{0}}+e^{-\mu t} H_{t_{0}} \int_{t_{0}}^{t} e^{\mu s} d b_{s}+e^{-\mu t} \int_{t_{0}}^{t}\left(H_{s}-H_{t_{0}}\right) e^{\mu s} d b_{s}
$$

Of the three terms on the right-hand side, the first tends to 0 as $t \rightarrow \infty$, the last term is 'small' when $t_{0}$ is large, and the middle term has the following form: $H_{t_{0}}$ times a variable independent of $H_{t_{0}}$ with the Gaussian $\mathrm{N}\left(0,\left\{1-e^{-2 \mu\left(t-t_{0}\right)}\right\} / 2 \mu\right)$ distribution. So, we have the following.

Heuristic IDEA 4.3.1. Conditionally on $M_{\infty}, e^{-\mu t} N_{t}$ has asymptotically the $\mathrm{N}\left(0,2 M_{\infty} / \mu\right)$ distribution.

The desired result for Theorem 1.5.1, which we are in the process of proving, establishes (when one takes $\alpha_{0}=\alpha_{1}=0$ ) the following.

Weaker Statement 4.3.2. As $t \rightarrow \infty$, the law of the pair $\left(M_{t}, e^{-\mu t} N_{t}\right)$ converges in the 'weak' topology to the law of $\left(M_{\infty}, \xi \sqrt{2 M_{\infty} / \mu}\right)$, where $\xi$ denotes a standard normal $\mathrm{N}(0,1)$ variable independent of $M_{\infty}$.

We now have

$$
\exp \left\{-x\left(\varphi_{t} s_{-t} z\right)\right\}=\mathbb{E}^{x} \exp \left\{-\left(\alpha_{0}+\beta_{0} i\right) M_{t}-\alpha_{1} e^{-\lambda_{0} t} X_{t} v-\beta_{1} i e^{-\mu t} N_{t}\right\} .
$$

Fix $x$ and $z$. We choose $t_{0}$ so large that, for $t \geqslant t_{0}$,

$$
\mathbb{E}^{x}\left|\alpha_{1} e^{-\lambda_{0} t} X_{t} v\right| \leqslant \frac{1}{3} \varepsilon, \quad \mathbb{E}^{x}\left|\alpha_{0}+\beta_{0} i\right|\left|M_{t}-M_{\infty}\right| \leqslant \frac{1}{6} \varepsilon .
$$

Then, for $t \geqslant t_{0}$,

$$
\left|\exp \left\{-x\left(\varphi_{t} s_{-t} z\right)\right\}-\mathbb{E}^{x} \exp \left\{-\left(\alpha_{0}+\beta_{0} i\right) M_{t_{0}}-\beta_{1} i e^{-\mu t} N_{t}\right\}\right| \leqslant \frac{2}{3} \varepsilon .
$$

Looking at (4.3.2), we now assume $t_{0}$ chosen so that, in addition,

$$
\beta_{1}^{2} \mathbb{E}\left\{\left(H_{s}-H_{t_{0}}\right)^{2}\right\} \leqslant \frac{1}{9} \varepsilon^{2} \quad\left(s \geqslant t_{0}\right),
$$

whence $\beta_{1} i$ times the final integral at (4.3.2) has $L^{2}$ norm at most $\frac{1}{9} \varepsilon^{2}$ and hence $L^{1}$ norm at most $\frac{1}{3} \varepsilon$. Hence, for $t \geqslant t_{0}$,

$$
\left|\exp \left\{-x\left(\varphi_{t} s_{-t} z\right)\right\}-\operatorname{expression}\right| \leqslant \varepsilon,
$$


where

expression

$$
\begin{aligned}
& =\mathbb{E}^{x} \exp \left\{-\left(\alpha_{0}+\beta_{0} i\right) M_{t_{0}}-\beta_{1} i e^{-\mu t} N_{t_{0}}-\beta_{1} i e^{-\mu t} H_{t_{0}} \int_{t_{0}}^{t} e^{\mu s} d b_{s}\right\} \\
& =\mathbb{E}^{x} \mathbb{E}^{x}\left(\cdot \mid \mathscr{F}\left(t_{0}\right)\right) \\
& =\mathbb{E}^{x} \exp \left\{-\left(\alpha_{0}+\beta_{0} i\right) M_{t_{0}}-\beta_{1} i e^{-\mu t} N_{t_{0}}-\frac{1}{2} \beta_{1}^{2} H_{t_{0}}^{2}\left(1-e^{-2 \mu\left(t-t_{0}\right)}\right) / 2 \mu\right\} .
\end{aligned}
$$

Hence, on letting $t \rightarrow \infty$ in (4.3.3), we see that any limit point of $\exp \left\{-x\left(\varphi_{t} s_{-t} z\right)\right\}$ lies within a distance of at most $\varepsilon$ of

$$
E^{x} \exp \left\{-\left(\alpha_{0}+\beta_{0} i\right) M_{t_{0}}-\frac{1}{2} \beta_{1}^{2} H_{t_{0}}^{2} / 2 \mu\right\} .
$$

But, as $t_{0} \rightarrow \infty$, this last expression converges to

$$
\mathbb{E}^{x} \exp \left\{-\left(\alpha_{0}+\beta_{0} i\right) M_{\infty}-\frac{1}{2} \beta_{1}^{2} 2 M_{\infty} / \mu\right\}=\Gamma\left(\left(\alpha_{0}+\beta_{0} i+\frac{1}{2} \beta_{1}^{2} 2 / \mu\right) u\right)=\Gamma(\Pi z) .
$$

The desired result follows.

The proof for the case when $\lambda_{0}=2 \lambda_{1}$ follows exactly the same lines as that just given for the case when $\lambda_{0}>2 \lambda_{1}$, the argument now involving

$$
\mathbb{E}_{\mathscr{F}\left(t_{0}\right)}^{x}\left(\exp \left\{-\beta_{1} i(1+t)^{-1 / 2} H_{t}\left(b_{t}-b_{t_{0}}\right)\right\}\right)=\exp \left(-\frac{1}{2} \beta_{1}^{2} H_{t_{0}}^{2} \frac{t-t_{0}}{1+t}\right) .
$$

Further details are omitted.

\subsection{Proof of the uniform-convergence result, Theorem 1.5.2}

Inevitably, this proof is achieved by combining compactness arguments with the pointwise-convergence result, Theorem 1.5.1, proved in the previous subsection. Several different compactness arguments, utilizing many of our previous results, are required.

It will be convenient to use the inner product and associated norm

$$
\langle z, w\rangle:=q_{2} \bar{z}_{1} w_{1}+q_{1} \bar{z}_{2} w_{2}, \quad\|z\|^{2}:=\langle z, z\rangle,
$$

with respect to which $Q$ is self-adjoint: $\langle z, Q w\rangle=\langle Q z, w\rangle$. Of course, $\|\cdot\|$ defines the standard Euclidean topology. The symbols $z$ and $w$ will henceforth always denote elements of $E$. We set

$$
N\left(e_{\infty}, \eta\right):=\left\{w:\left\|w-e_{\infty}\right\|<\eta\right\}:=\left\{w \in E:\left\|w-e_{\infty}\right\|<\eta\right\} .
$$

Lemma 4.4.1. The point $e_{\infty}$ is a sink for $\varphi$ in that the stability matrix for the linearization of $\varphi$ at $e_{\infty}$ has strictly negative real eigenvalues. For some $\eta_{0}>0$, we shall have

$$
\varphi_{t} N\left(e_{\infty}, \eta\right) \subseteq N\left(e_{\infty}, \eta\right) \quad \text { whenever } t \geqslant 0 \text { and } 0<\eta \leqslant \eta_{0} .
$$

Proof. With $e$ as shorthand for $e_{\infty}$, we have

$$
\left(r_{1}-q_{1}-2 e_{1}\right) e_{1}+q_{1} e_{2}=0, \quad\left(r_{2}-q_{2}-2 e_{2}\right) e_{2}+q_{2} e_{1}=0,
$$


and the stability matrix at $e$ is

$$
\begin{aligned}
R+Q-4 D(e) & =\left(\begin{array}{cc}
r_{1}-q_{1}-4 e_{1} & q_{1} \\
q_{2} & r_{2}-q_{2}-4 e_{2}
\end{array}\right) \\
& =\left(\begin{array}{cc}
-q_{1} e_{2} / e_{1}-2 e_{1} & q_{1} \\
q_{2} & -q_{2} e_{1} / e_{2}-2 e_{2}
\end{array}\right),
\end{aligned}
$$

which obviously has positive determinant and negative trace. The matrix is self-adjoint relative to $\langle\cdot, \cdot\rangle$. Hence, the two eigenvalues are real and strictly negative.

Let $z \in E$ and let $\zeta_{t}$ denote $\varphi_{t} z-e_{\infty}$. Then

$$
\left.\frac{d}{d t}\left(\left\|\zeta_{t}\right\|^{2}\right)\right|_{t=0}=2\left\langle\zeta_{0},(R+Q-4 D(e)) \zeta_{0}\right\rangle+\text { 'cubic terms' in } \zeta_{0},
$$

and, since $R+Q-4 D(e)$ is negative-definite, then, for suitably small $\eta_{0}$, the derivative at (4.4.2) is negative for all $z \in N\left(e_{\infty}, \eta_{0}\right) \backslash\left\{e_{\infty}\right\}$.

Lemma 4.4.2. Let $r>0$ and $0<\varepsilon<\eta_{0}$. Then there exists $t_{0}(r, \varepsilon)$ such that

$$
\varphi_{t}(z) \in N\left(e_{\infty}, \varepsilon\right) \quad \text { whenever } t \geqslant t_{0}(r, \varepsilon) \text { and }\|z\| \geqslant r \text {. }
$$

Proof. First choose $t_{1}$ so that $e_{t_{1}} \in N\left(e_{\infty}, \frac{1}{2} \varepsilon\right)$. Since $\varphi_{t_{1}} z \rightarrow e_{t_{1}}$ as $z \rightarrow \infty_{E}$, we can choose $R_{1}$ so that $\varphi_{t_{1}} z \in N\left(e_{\infty}, \varepsilon\right)$ whenever $\|z\| \geqslant R_{1}$. Now let

$$
A:=\left\{z: r \leqslant\|z\| \leqslant R_{1}\right\} .
$$

Assume that $A$ is non-empty, or there is nothing further to prove. Now $A$ is compact. For each $z \in A$, we have $\varphi_{t} z \rightarrow e_{\infty}$ as $t \rightarrow \infty$; so we can choose $\tau(z)$ such that $\varphi_{\tau(z)} z \in N\left(e_{\infty}, \frac{1}{2} \varepsilon\right)$. By continuity of $\varphi_{\tau(z)}(\cdot)$, there will exist a neighbourhood (in $A) G(z)$ of $z$ such that $\varphi_{\tau(z)}(w) \in N\left(e_{\infty}, \varepsilon\right)$ for $w \in G(z)$. A finite collection $G\left(z_{1}\right), G\left(z_{2}\right), \ldots, G\left(z_{n}\right)$ of these neighbourhoods covers $A$. We now have the desired result with

$$
t_{0}(r, \varepsilon)=\max \left(t_{1}, \tau\left(z_{1}\right), \ldots, \tau\left(z_{n}\right)\right) .
$$

LEMMA 4.4.3. For $r>0$,

$$
\begin{gathered}
m(r):=\inf \left\{\left\|\varphi_{t}(z)\right\|: t \geqslant 0,\|z\|=r\right\}>0, \\
\quad \inf \left\{\left\|\varphi_{t}(z)\right\|: t \geqslant 0,\|z\| \geqslant r\right\}=m(r) .
\end{gathered}
$$

Proof. Let $0<\eta<\left\|e_{\infty}\right\|$. Then, by Lemma 4.4.2,

But

$$
\inf \left\{\left\|\varphi_{t}(z)\right\|: t \geqslant t_{0}(r, \eta),\|z\|=r\right\}>\left\|e_{\infty}\right\|-\eta>0 .
$$

$$
\left\{\varphi_{t}(z): t \leqslant t_{0}(r, \eta),\|z\|=r\right\}
$$

is compact and does not contain $(0,0)$. Result (4.4.3) follows, and result (4.4.4) is then clear from the flow property.

Lemma 4.4.4. For any compact subset $F$ of $E$, the convergence

$$
\varphi_{t} s_{-t} z \rightarrow \Gamma \Pi z \quad(t \rightarrow \infty)
$$

is uniform in $z \in F$. 
Proof. For each $x$,

$$
\exp \left\{-x\left(\varphi_{t} s_{-t} z\right)\right\} \rightarrow \exp \{-x(\Gamma \Pi z)\}
$$

each side having the form of a Fourier-Laplace transform (FLT)

$$
\mathbb{E}^{x} \exp \{-Y(z)\}
$$

of an $\mathscr{M}(I)$-valued (hence $\left(\mathbb{R}^{+}\right)^{2}$-valued) random variable $Y$. However, it is a standard result that if a sequence of FLTs of $\left(\mathbb{R}^{+}\right)^{2}$-valued random variables converges pointwise on $E$ to another such FLT, then it must do so uniformly on compact subsets of $E$. This follows by combining the 'tightness' property of 'weak'-convergence theory with the estimate (4.3.1). Now read Note 3.2.2.

LEMMA 4.4.5. For $r>0$,

$$
c(r):=\inf \left\{\left\|\varphi_{t} s_{-t} z\right\|: t \geqslant 0,\|z\|=r\right\}>0 .
$$

Proof. The set $\{\Gamma \Pi z:\|z\|=r\}$ is compact, and does not contain $(0,0)$. On combining this with Lemma 4.4.4, we find that for some $t_{2}$,

$$
\inf \left\{\left\|\varphi_{t} s_{-t} z\right\|: t \geqslant t_{2},\|z\|=r\right\}>0 .
$$

However, $\left\{\varphi_{t} s_{-t} z: t \leqslant t_{2},\|z\|=r\right\}$ is compact, and does not contain $(0,0)$. The result follows.

LEMMA 4.4.6. For $r>0$,

$$
d(r):=\inf \left\{\left\|\varphi_{t} s_{-t} z\right\|: t \geqslant 0,\|z\| \geqslant r\right\}>0 .
$$

We are first going to prove Lemma 4.4.6 and Theorem 1.5.2 when $\lambda_{0} \neq 2 \lambda_{1}$, and then we shall prove these two results when $\lambda_{0}=2 \lambda_{1}$.

Case 1. Suppose for the time being that $\lambda_{0} \neq 2 \lambda_{1}$. Then (look at the definition!)

$$
\left\{s_{-t}: t \geqslant 0\right\} \text { has the flow property. }
$$

(One can think of $\mathbb{C}^{2}$ as a vector space over $\mathbb{R}$ with basis $u, i u, v, i v$.)

Proof of Lemma 4.4.6 when $\lambda_{0} \neq 2 \lambda_{1}$. Suppose that $\|z\| \geqslant r$ and that $t \geqslant 0$. It may happen that $\left\|s_{-t_{3}} z\right\|=r$ for some $t_{3} \leqslant t$, and then

$$
\varphi_{t} s_{-t} z=\varphi_{t_{3}} \varphi_{t-t_{3}} s_{-\left(t-t_{3}\right)} s_{-t_{3}} z=\varphi_{t_{3}}\left(z^{\prime}\right),
$$

where $\left\|z^{\prime}\right\| \geqslant c(r)$ and so $\left\|\varphi_{t} s_{-t} z\right\| \geqslant m(c(r))$. Otherwise, it must be the case that $\left\|s_{-t} z\right\| \geqslant r$ and then $\left\|\varphi_{t} s_{-t} z\right\| \geqslant m(r)$. The desired result follows with $d(r):=m(r) \wedge m(c(r))$.

Proof of Theorem 1.5.2 when $\lambda_{0} \neq 2 \lambda_{1}$. Let $\varepsilon>0$ be given. Choose $t_{4}$ so that

$$
t_{4} \geqslant t_{0}\left(d(1), \frac{1}{2} \varepsilon\right), \quad\left\|\Gamma \Pi z-e_{\infty}\right\|<\frac{1}{2} \varepsilon \text { whenever }\left\|s_{-t_{4}} z\right\|>1 .
$$

Then, for $t \geqslant t_{4}$ and $\left\|s_{-t_{4}} z\right\|>1$, we have

$$
\varphi_{t} s_{-t} z=\varphi_{t_{4}} \varphi_{t-t_{4}} s_{-\left(t-t_{4}\right)} s_{-t_{4}} z=\varphi_{t_{4}}\left(z^{*}\right),
$$


where $\left\|z^{*}\right\| \geqslant d(1)$, so that

$$
\left\|\varphi_{t} s_{-t} z-e_{\infty}\right\|=\left\|\varphi_{t_{4}} z-e_{\infty}\right\|<\frac{1}{2} \varepsilon .
$$

Thus, for $\left\|s_{-t_{4}} z\right\|>1$ and $t \geqslant t_{4}$,

$$
\left\|\varphi_{t} s_{-t} z-\Gamma \Pi z\right\|<\varepsilon .
$$

Since $\varphi_{t} s_{-t} z \rightarrow \Gamma \Pi z$ uniformly on the compact set $\left\{z:\left\|s_{-t_{4}} z\right\| \leqslant 1\right\}$, the proof of Theorem 1.5.2 when $\lambda \neq 2 \lambda_{1}$ is complete.

Case 2. Now suppose that $\lambda_{0}=2 \lambda_{1}$. The maps $\left\{s_{-t}: t \geqslant 0\right\}$ no longer constitute a flow. For $0 \leqslant r \leqslant t$, we have

$$
s_{-t}=s_{-(t-r)} q_{-(t-r),-t},
$$

where $q_{-(t-r),-t}$ is the $\mathbb{R}$-linear map with

$$
q_{-(t-r),-t} w= \begin{cases}s_{-r} w & \text { if } w=u, i u, \text { or } v \\ \left(c_{-(t-r),-t}\right)^{1 / 2} s_{-r} w & \text { if } w=i v\end{cases}
$$

where

$$
c_{-(t-r),-t}=\frac{(1+t-r)(1+r)}{1+t}=1+\frac{(t-r) r}{1+t} \geqslant 1,
$$

and indeed, we have

$$
\left\|q_{-(t-r),-t} w\right\| \geqslant\left\|s_{-r} w\right\| \quad \text { for all } w \in E \text { and pairs }(r, t) \text { with } 0 \leqslant r \leqslant t .
$$

The last observation uses (for the 'imaginary parts') the fact that $u$ and $v$ are orthogonal relative to our new inner product at (4.4.1).

Proof of Lemma 4.4.6 when $\lambda_{0}=2 \lambda_{1}$. This proof is an obvious modification of the earlier one. Suppose that $\|z\| \geqslant r$ and that $t \geqslant 0$. It may happen that $\left\|q_{-\left(t-t_{3}\right), t} z\right\|=r$ for some $t_{3} \leqslant t$, and then

$$
\varphi_{t} s_{-t} z=\varphi_{t_{3}} \varphi_{t-t_{3}} s_{-\left(t-t_{3}\right)} q_{-\left(t-t_{3}\right), t} z=\varphi_{t_{3}}\left(z^{\prime}\right),
$$

where, as before, $\left\|z^{\prime}\right\| \geqslant c(r)$ and so $\left\|\varphi_{t} s_{-t} z\right\| \geqslant m(c(r))$. Otherwise, it must be the case that $\left\|s_{-t} z\right\| \geqslant r$ (yes, this is the same as before!) and then $\left\|\varphi_{t} s_{-t} z\right\| \geqslant m(r)$. The desired result again follows with $d(r):=m(r) \wedge m(c(r))$.

Proof of Theorem 1.5.2 when $\lambda_{0}=2 \lambda_{1}$. Because of (4.4.7), this is now identical to the proof for the case when $\lambda_{0} \neq 2 \lambda_{1}$ except for one line where we modify (4.4.6) in the way we modified (4.4.5) to (4.4.8).

Acknowledgements. This paper was written at Bath. We thank the members of Bath's Reaction-Diffusion Group, and especially John Toland and Francis Burstall, for many helpful comments. JW was supported by EPSRC Grant GR/K70397 at Bath.

The remarks of an extremely conscientious and perspicacious referee have greatly improved this paper.

\section{References}

1. V. I. ARnold, Geometric methods in the theory of ordinary differential equations (translated from the Russian, Springer, Berlin, 1983). 
2. K. B. Athreya and P. E. Ney, Branching processes (Springer, Berlin, 1972).

3. M. D. Bramson, 'Maximal displacement of branching Brownian motion', Comm. Pure. Appl. Math. 31 (1978) 531-581.

4. M. D. Bramson, 'Convergence of the solutions of the Kolmogorov equation to travelling waves', Mem. Amer. Math. Soc. 285 (1983) 1-190.

5. D. A. Dawson, 'Measure-valued Markov processes', École d'Été de Probabilités de Saint-Flour XXI, 1993 (ed. P. L. Henniquin), Lecture Notes in Mathematics 1541 (Springer, Berlin, 1993) $2-260$.

6. E. Hille, Ordinary differential equations in the complex domain (Wiley, New York, 1976).

7. L. HöRMANDER, The analysis of linear partial differential operators, III (Springer, Berlin, 1984).

8. H. P. McKean, 'Application of Brownian motion to the equation of Kolmogorov-PetrovskiiPiskunov', Comm. Pure Appl. Math. 28 (1975) 323-331.

9. J. Neveu, 'Multiplicative martingales for spatial branching processes', Seminar on Stochastic Processes (ed. E. Çinlar, K. L. Chung and R. K. Getoor), Progress in Probability and Statistics 15 (Birkhäuser, Boston, 1987) 223-241.

10. D. Revuz and M. Yor, Continuous martingales and Brownian motion (Springer, Berlin, 1991).

11. L. C. G. Rogers and D. Williams, Diffusions, Markov processes and martingales. Volume 1: foundations, 2nd edn (Wiley, Chichester, 1994).

12. L. C. G. Rogers and D. Williams, Diffusions, Markov processes and martingales. Volume 2: Itô calculus (Wiley, Chichester, 1987).

13. J. WARren, 'Some aspects of branching processes', PhD Thesis, Bath University, 1995.

\section{Jon Warren \\ Department of Statistics \\ University of Warwick \\ Coventry CV4 7AL}

J.Warren@warwick.ac.uk
David Williams

Department of Mathematics

University of Wales, Swansea

Singleton Park

Swansea SA2 8PP 